\title{
Safeguards Envelope Progress FY08
}

Richard Metcalf

Aaron Bevill

Robert Bean

September 2008

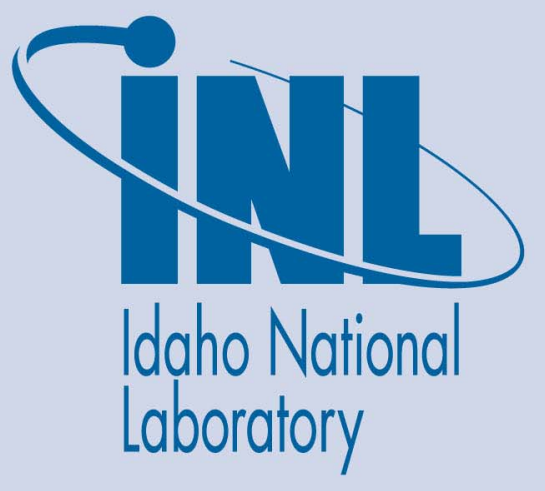

The INL is a U.S. Department of Energy National Laboratory operated by Battelle Energy Alliance 


\title{
Safeguards Envelope Progress FY08
}

\author{
Richard Metcalf \\ Aaron Bevill \\ Robert Bean
}

September 2008

\section{Idaho National Laboratory \\ Idaho Falls, Idaho 83415}

http://www.inl.gov

Prepared for the

U.S. Department of Energy

Office of Nuclear Energy

Under DOE Idaho Operations Office

Contract DE-AC07-05ID14517 


\title{
Safeguards Envelope Progress FY08
}

\author{
INL/EXT-08-14915
}

Revision 0

September 2008

Approved by

Robert Bean

$10 / 8 / 2008$ 


\section{EXECUTIVE SUMMARY}

The Safeguards Envelope Project met its milestones by creating a rudimentary safeguards envelope, proving the value of the approach on a small scale, and determining the most appropriate path forward. The Idaho Chemical Processing Plant's large cache of reprocessing process monitoring data, dubbed Undeniably Big Extraction of Reprocessing Data (UBER Data), was recovered and used in the analysis. A probabilistic $Z$ test was used on a Markov Monte Carlo simulation of expected diversion data when compared with normal operating data. The data regarding a fully transient event in a tank was used to create a simple requirement, representative of a safeguards envelope, whose impact was a decrease in operating efficiency by $1.3 \%$ but an increase in material balance period of $26 \%$. This approach is operator, state, and international safeguards friendly and should be applied to future reprocessing plants. Future requirements include tank-to-tank correlations in reprocessing facilities, detailed operations impact studies, simulation inclusion, automated optimization, advanced statistics analysis, and multi-attribute utility analysis.

\section{FOREWORD}

The work described herein is under the sponsorship of the U. S. Department of Energy Safeguards Campaign Modeling and Simulation Crosscut, with additional support from the DOE/NNSA Office of International Regimes and Agreements.

\section{ACKNOWLEDGEMENTS}

Neil Liester of Idaho Cleanup Project helped recover the best process monitoring data in the world, without which we would have wasted thousands of man hours. Mike Ehinger of Oak Ridge National Laboratory deserves a hand-shake for the sheer volume

of knowledge he has imparted to the authors. Mike Zentner of Pacific Northwest National Laboratory has been the best devil's advocate a team could ask for. Tom Burr of Los Alamos National Lab was a critical help and his early works were an inspiration.

Jeffery Jay of Savannah National Laboratory also deserves a nod for his help in acquiring data. Additionally, Jack Law of Idaho National Laboratory was a great help in understanding what factors we should consider. Finally, Richard and Aaron would like to thank Jean Ragusa and William Charlton of Texas A\&M, their steadfast advisors. 


\section{CONTENTS}

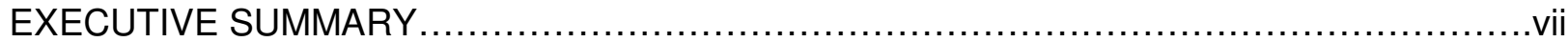

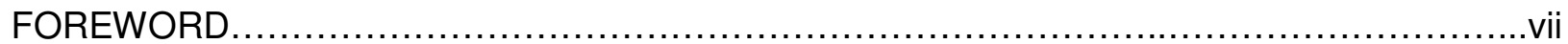

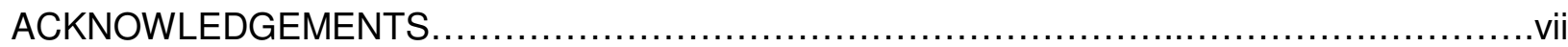

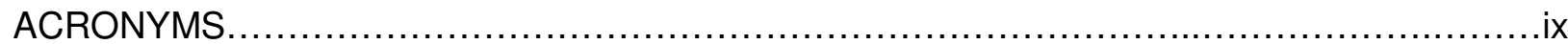

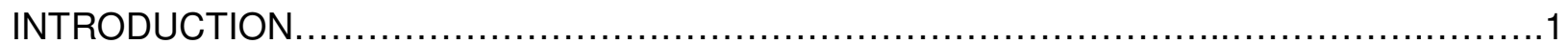

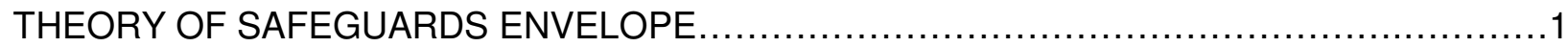

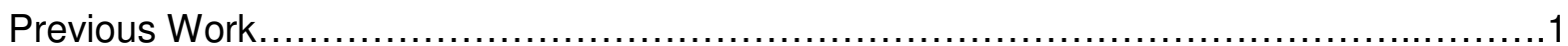

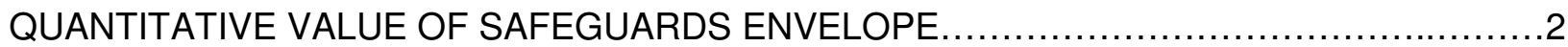

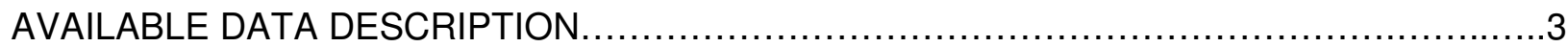

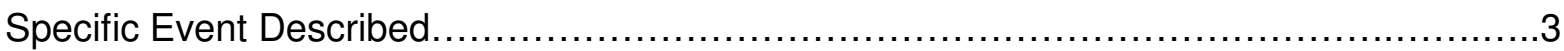

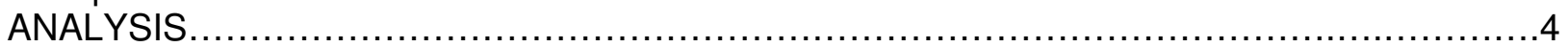

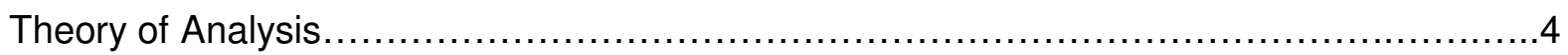

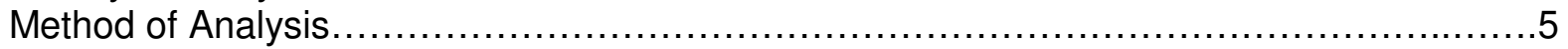

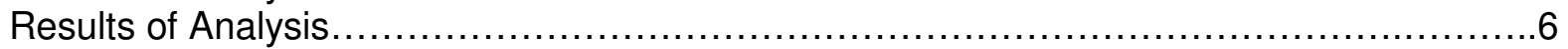

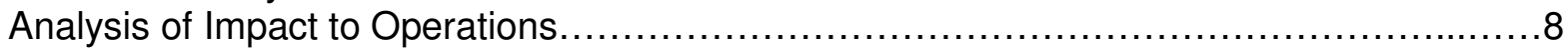

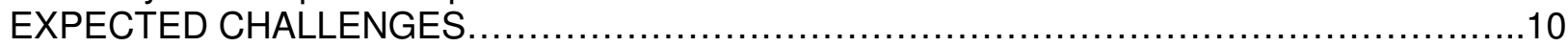

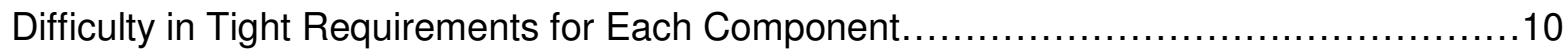

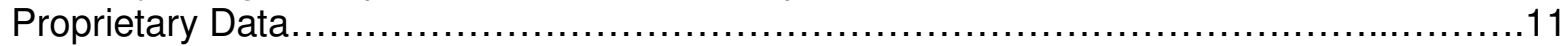

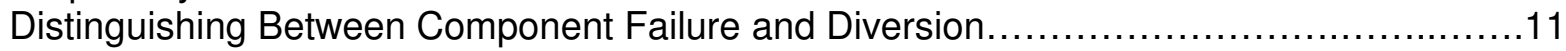

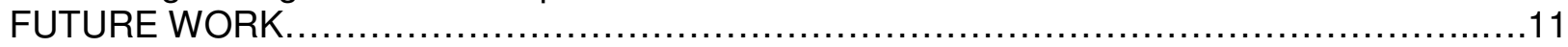

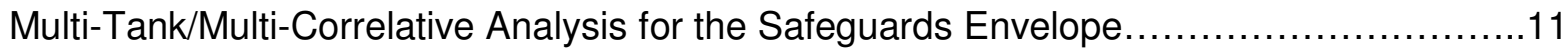

Multi-Attribute Utility Analysis for Barriers to Proliferation..................................

Multi-Attribute Utility Analysis for Diversion Detection...................................

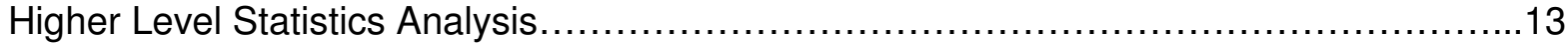

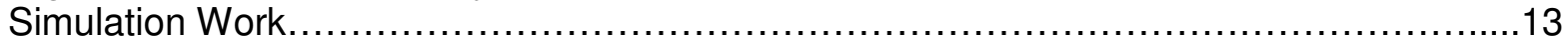

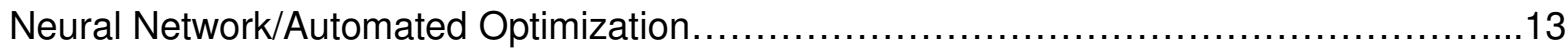

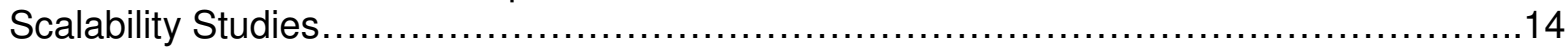

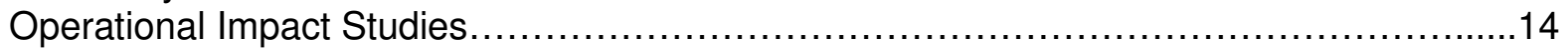

APPENDIX A: Detailed Analysis of Process Monitoring Benefit.............................15 APPENDIX B: Simulation and Optimization of Process Monitoring Safeguards by Semi-

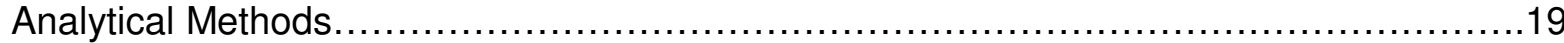

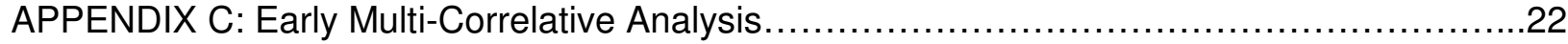

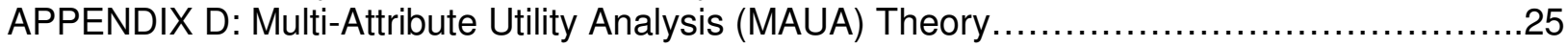

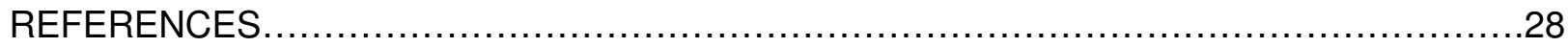




\section{ACRONYMS}

$\begin{array}{ll}\text { DOE } & \text { Department of Energy } \\ \text { FAR } & \text { false alarm rate } \\ \text { GIF } & \text { GEN IV International Forum } \\ \text { ICPP } & \text { Idaho Chemical Processing Plant } \\ \text { IAEA } & \text { International Atomic Energy Agency } \\ \text { MBP } & \text { material balance period } \\ \text { MC\&A } & \text { material control and accountability } \\ \text { MUF } & \text { material unaccounted for } \\ \text { MUFAT } & \text { material unaccounted for alarm threshold } \\ \text { MAUA } & \text { multi-attributes utility analysis } \\ \text { NRTA } & \text { near real time accountancy } \\ \text { NRC } & \text { Nuclear Regulatory Commission } \\ \text { ORNL } & \text { Oak Ridge National Laboratory } \\ \text { PP } & \text { physical protection } \\ \text { PND } & \text { probability of nondetection } \\ \text { PM } & \text { process monitoring } \\ \text { SNM } & \text { special nuclear material } \\ \text { SQ } & \text { special quantity } \\ \text { TIDs } & \text { tamper indicating devices } \\ \text { UBER DATA } & \text { Undeniably Big Extraction of Reprocessing Data }\end{array}$




\section{INTRODUCTION}

The future expansion of nuclear power will require not just electricity production but fuel cycle facilities such as fuel fabrication and reprocessing plants. As large reprocessing facilities are built around the world, they must be built and operated in a manner which minimizes the risk of nuclear proliferation. Increased detection capability, the workhorse of the safeguards community, has met the challenges so far but at significant cost. Future approaches will need to utilize safeguards by design as well as maximizing the value from all data available at a reprocessing facility. One of these future approaches, the Safeguards Envelope, is realizable in the near future with significant benefits.

\section{THEORY OF SAFEGUARDS ENVELOPE}

A Safeguards Envelope is a set of operational parameters that maximize detection by safeguards systems and inventory balance period while minimizing false positive rates and operator impact. The Safeguards Envelope concept is based on the successful Safety Envelope method, discussed in a previous paper. ${ }^{1}$ A Safeguards Envelope is a natural expansion of currently proposed research in safeguards by design, near real time accountancy (NRTA), and integrated safeguards approaches.

\section{Previous Work}

There are three major proposed areas of influence for the Safeguards Envelope approach: Physical Protection (PP), Material Control and Accountability (MC\&A), and Process Monitoring (PM). Though PP and MC\&A are expected to be integrated with $\mathrm{PM}$, the well-established theory and application of both PP and MC\&A requires that this research must show integration of newer technology into the accepted methods. Therefore, the research this year focused on demonstration of a safeguards envelope with regard to PM-based safeguards in addition to existing MC\&A and PP systems. The most relevant previous work was in process monitoring for safeguards. There were three major previous works considered in detail: the Integrated Equipment Test Facility's Oak Ridge National Laboratory (ORNL) PM system evaluated in 1986, the diversion detection methods developed by Thomas Burr at Los Alamos National Laboratory using FACSIM, and the higher level statistics analyses of various authors. ${ }^{2} 3$ 45678910

Most PM methods to date rely on the analysis of cumulative residuals in order to determine divergence from an expected value. Even modern learning algorithms either rely on some existing modeling and then use PM to correct the existing models or are formed from months of previous operating data. Previous work has shown that large scale PM is possible. No previous research, however, appears to have considered exchanging a small operator impact for a large increase in PM detection capability. 


\section{QUANTITATIVE VALUE OF SAFEGUARDS ENVELOPE}

When supplementing accounting methods with PM security measures, either of two systems may be applied. In the first model (union model), either alarm may warrant an investigation. Alternatively, monitors may choose to only investigate cases in which both alarms sound (intersection model). Since both alarms must sound to warrant investigation under the intersection model, a very sensitive PM system is needed.

Sensitivity and specificity are not intrinsic properties of a monitoring system; rather, the monitoring system only relates the two. For example, both $\alpha_{A}$, the probability of nondetection (PND) of accountancy and $\beta_{A}$, the false alarm rate (FAR) of accountancy depend on the material unaccounted for (MUF) alarm threshold. Thus, for a given $\alpha_{P M}$, it is possible to compute $\beta_{P M}$ such that the overall system sensitivity and specificity remain the same. This represents a threshold for the usefulness of a PM detection system: any system producing a higher FAR than the threshold will only interfere with plant operation and any system with a higher PND poses an unacceptable risk.

Additionally, for any PM method with the FAR below threshold, we can estimate the allowable standard deviation in accountancy measurements and estimate the allowable material balance period (MBP), assuming the standard deviation of measurements is proportional to the MBP. A detailed explanation of this style of analysis is given in Appendix A

Figures $1 \mathrm{a}$ and $1 \mathrm{~b}$ show the results, using the assumption of an eight-day MBP.

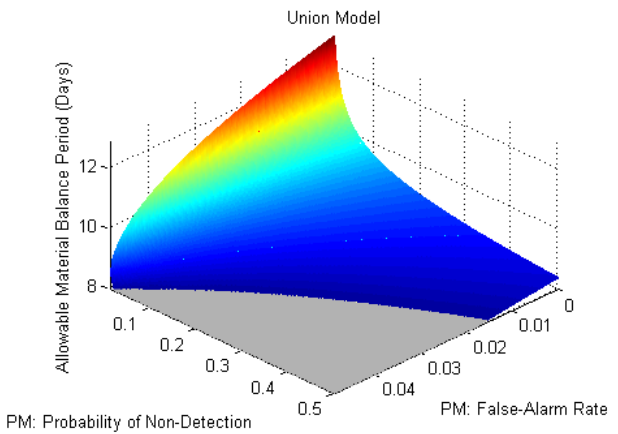

(a)

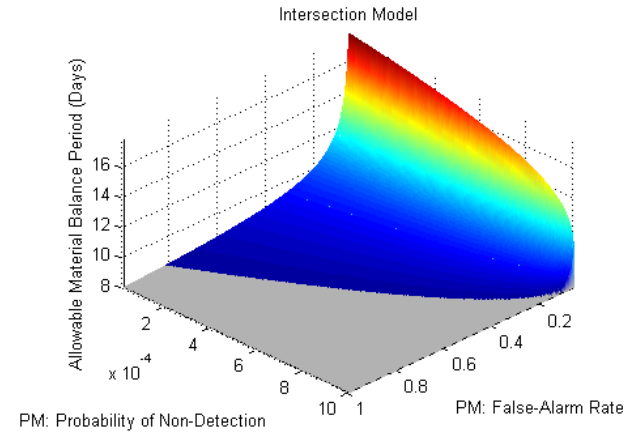

(b)

Figures $1 \mathrm{a}$ and $1 \mathrm{~b}$. Maximum allowable MBP for given PM characteristics. Note that the gray (lower) surface represents a region below the threshold of benefit.

The allowable MBP grows without bounds as $\beta_{\mathrm{PM}}$ approaches $0.01 \%$ for the union model or a approaches $5 \%$ for the intersection model. Of course, if one can reach an acceptable level of confidence strictly by PM, accounting becomes unnecessary. It is not this paper's contention that only using PM safeguards are cost-effective or even possible; the method contained simply represents a function to test the usefulness of 
PM analysis or analyze the most effective way to set PM thresholds to reduce the burden on accounting measurements.

\section{AVAILABLE DATA DESCRIPTION}

Locating reprocessing plant data that is measured and recorded at the intervals required to apply PM methods and create a Safeguards Envelope has been difficult. It was originally intended to simulate diversions using an existing model, but no model was found that had been verified and validated which could provide transient data of the type typically measured in reprocessing plants. Institutional archaeology yielded a significant amount of data from the Idaho Chemical Processing Plant (ICPP). This facility intended to have remote monitoring at a point and so had a significant amount of digital data collection. At its peak, the ICPP measured 1500 points in the process every minute (recorded every 4 minutes). The ICPP recorded data from 1986 to 2004 . Typical measurements included floating point numbers such as temperature, density, and tank level as well as binary points such as jet activation and safeguards tamper indicating devices (TIDs). This data set, the Undeniably Big Extraction of Reprocessing Data (UBER Data), is currently being recovered in full under a grant from the National Nuclear Security Administrations Office of International Safeguards.

\section{Specific Event Described}

In order to remain relevant, a transient event was isolated. Static tank anomaly detection has been covered thoroughly in the previous work, but a fully transient situation has yet to be demonstrated. Event \#1, shown in Figure 2, is a fully transient event extracted from the UBER Data. At no point does the tank reach a steady state from between the points at which it is empty. Event \#1 is for a tank with the measurement given in percentage of tank that was recorded once every four minutes. In this event, a tank was filled to near capacity and then began emptying immediately. This presents a unique challenge because a diversion taking place over this event would be hidden in the movement of the material and after the tank is completely empty, the systematic errors between two tanks may make it difficult to determine that material was removed. 


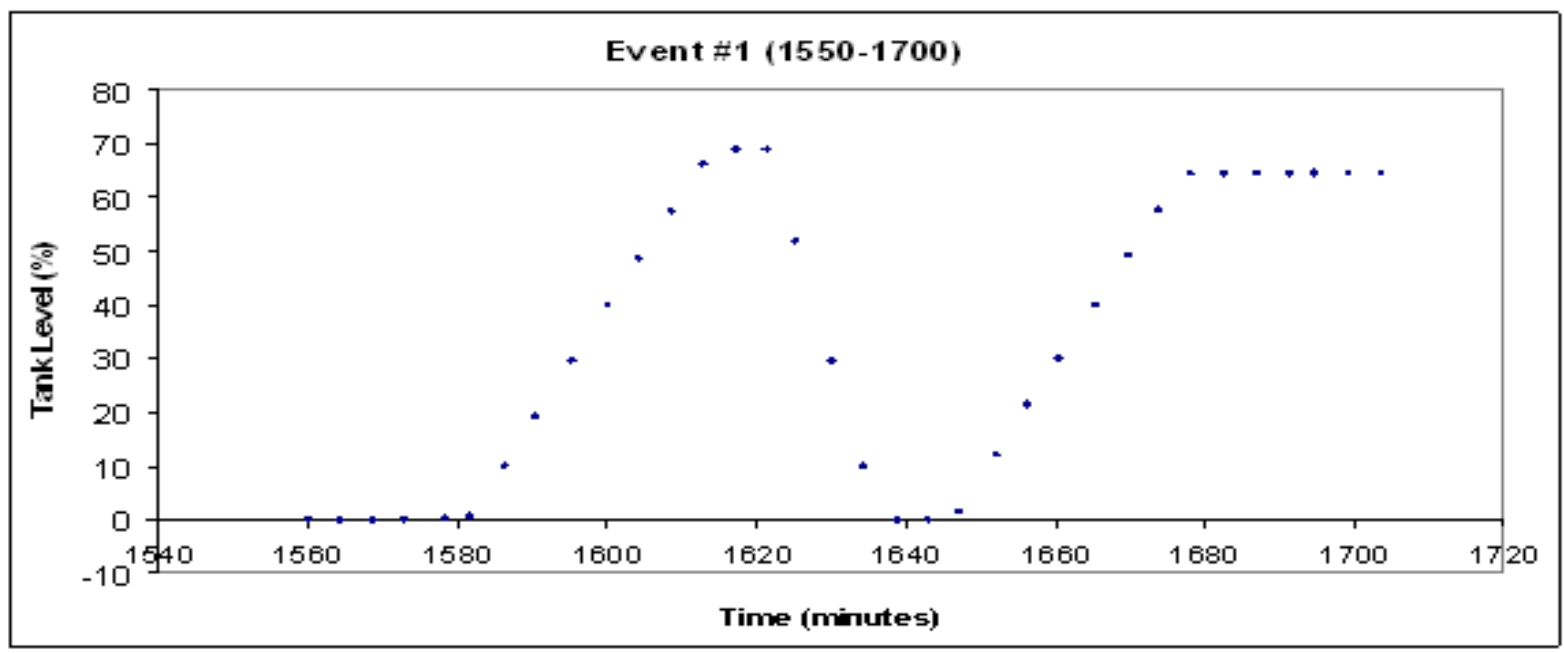

Figure 2. Event \#1 is a fully transient event between 1580 and 1640 minutes.

\section{ANALYSIS}

\section{Theory of Analysis}

The anomaly detection method that is being used is based on residual analysis, building on the previous research. Only the raw data is available and so an appropriate level of noise must be added for every "measurement" to be considered in the analysis.

At a minimum, three value sets must be considered: a "true" value set, a "normal operation" value set, and a "diversion" set. "True" values may be based on a simulation, analytic model, or empirical evidence. These true values are not correct values because there will always be a level of uncertainty which must be considered. The normal operation set can simply be the appropriate level of statistical noise based on sensor type added to the known actual data. The diversion set of data should have a certain value of tank level stolen from the correct values and then statistical noise added. The method that will be used for detecting the diversion is most vulnerable to a constant removal. The statistical noise of the "true" value set should be significantly less than the other sets if the model is well known or there is significant empirical evidence but could be more if only simulation exists.

These three sets can then be compared to determine the PND and FAR by simple probabilistic Z or students' t-tests. It appears as if the simplest method for determining the PND and FAR is a Markov Monte Carlo approach. A second considered approach which may be of more value as computational power increases is a semi-analytic approach, described in Appendix B. In this situation, the statistical noise is added randomly to every point along the transient. An alarm is triggered if a case exceeds a threshold determined by the expected deviation of the normal set and optimized to minimize both PND and FAR. This threshold optimization can be done by running several thousand cases of a diversion set and normal set against the "true" values. The percentage of diversion sets which fails to be detected becomes PND and the 
percentage of normal sets falsely detected to be a diversion constitutes the FAR. Figure 3 shows this procedure graphically.

Z-tests

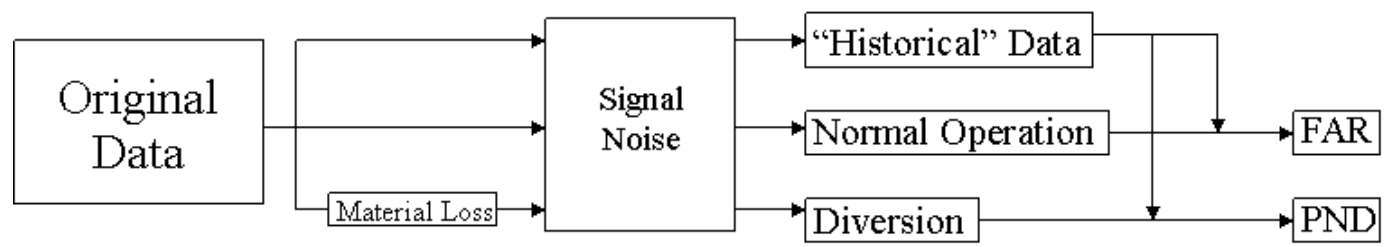

Figure 3. Procedure for basic PM comparison.

This analysis is one of the first demonstrated over transient data, but it does not represent a significant contribution to the theory of the Safeguards Envelope alone. In order to simulate the effects of a Safeguards Envelope, a minor alteration to operations in order to drastically increase detection probability, three other sets of data were created. These additional sets are the same statistical noise applied on points interpolated between the known data points. This is intended to be representative of an operator performing transient modes half as quickly or doubling the number of measurements under the Safeguards Envelope requirement of "When filling or emptying a tank, remove at half maximum speed." Figure 4 shows the Safeguards Envelope operation parameters.

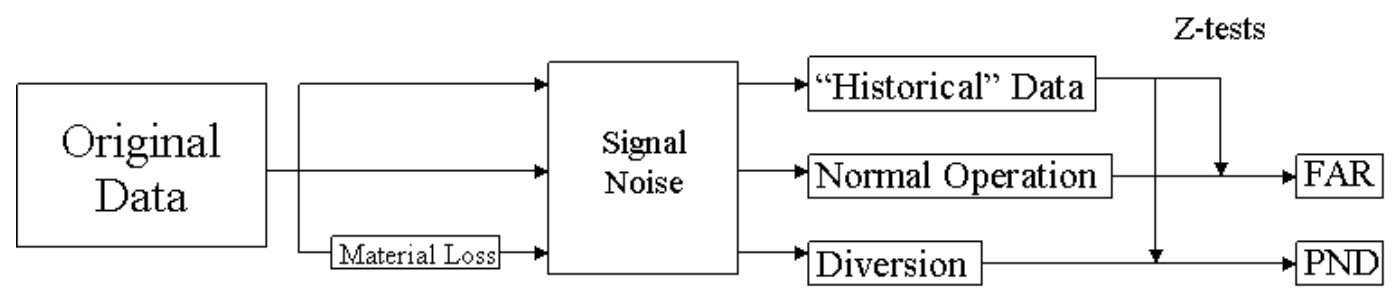

Figure 4. Safeguards Envelope operation should minimize FAR and PND.

The goal is to demonstrate significantly decreased FAR and PND based on Safeguards Envelope operation. Only a significant decrease in FAR and PND can justify the operational interference. It is important to note that the threshold for Safeguards Envelope operation should be different than normal operation and this requires a full new set of Markov simulations. In all cases, it is unacceptable to increase the FAR while reducing the PND because this creates a significant burden on the International Atomic Energy Agency (IAEA) internationally or Nuclear Regulatory Commission (NRC) and Department of Energy (DOE) domestically. It is also unacceptable for the PND to rise above the current level as that is a regulatory requirement.

\section{Method of Analysis}

Event \#1 is considered in the analysis. Since the material in Event \#1 is normalized in percentage and the amount of special nuclear material (SNM) in each percent is not explicitly stated, it was assumed that $0.5 \%$ of the tank would be stolen over the course of the transient (roughly an hour). An exaggerated example of this style of theft is shown in Figure 5. Since the errors associated with the measurements fell well below IAEA 
upper limits (and therefore may not represent data available at commercial plants), synthetic Gaussian noise was added to the measurements based IAEA measurement Target Values. ${ }^{11}$

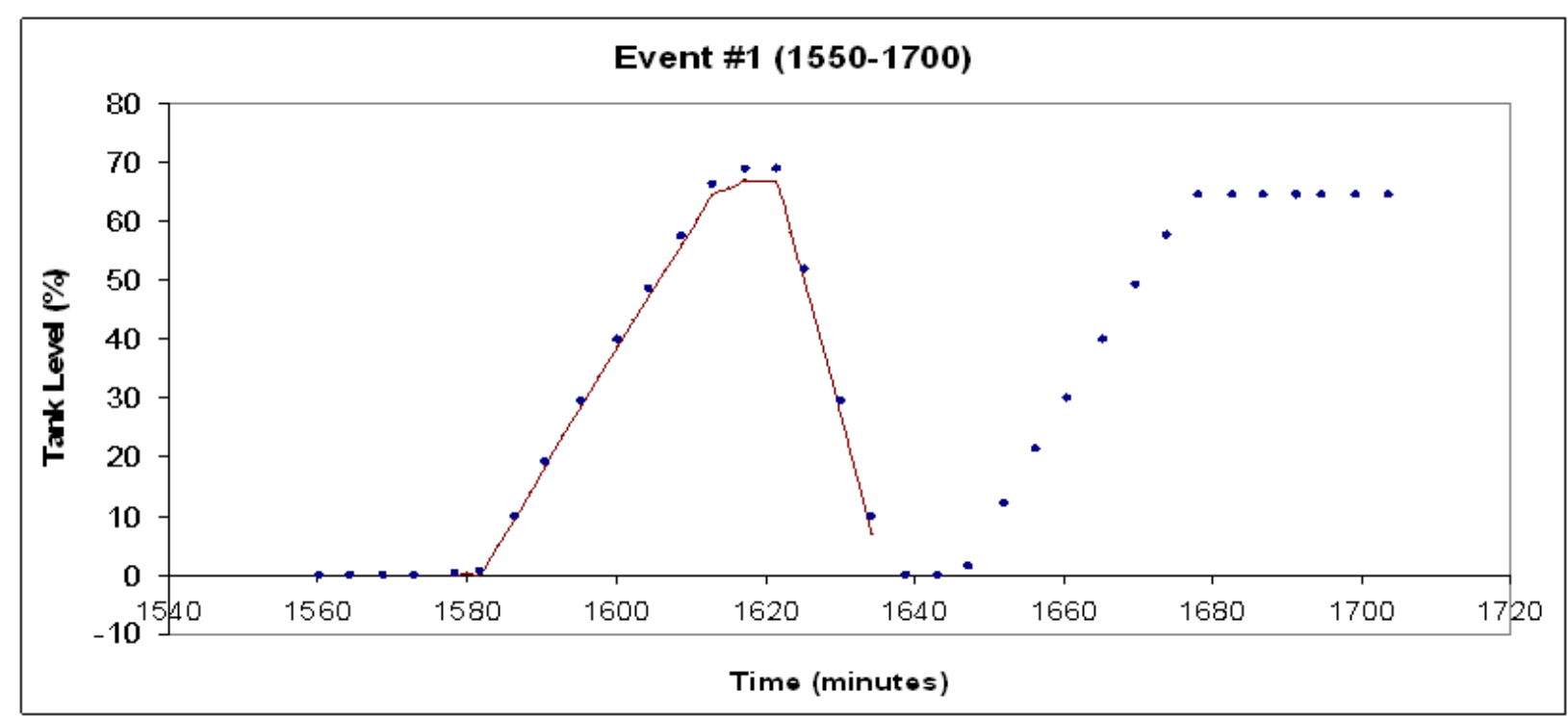

Figure 5. This example steals a total of $3 \%$ over the transient to show how the diversion is expected to occur. A theft of $0.5 \%$ is indistinguishable by the naked eye and so the theft is exaggerated for this figure.

$Z$ tests were performed at every point after the seventh, such that if any point would trigger an alarm, the entire event was triggered for investigation. The thresholds for the normal operation and Safeguards Envelope operation were optimized independently. To determine the PND and FAR, two thousand cases were run for each of the tested thresholds which spanned from 0 to 0.5 (unit less). The optimized threshold was eventually measured to a confidence $\pm 5 \mathrm{E}-8$ after several iterations at lower fidelity.

In order to be conservative, several assumptions were made. IAEA target values were used instead of the significantly (almost an order of magnitude) lower actual variance determined from steady state analysis. Even though all points were measured every minute, they were only recorded every four minutes. Basic $Z$ testing was used instead of several possible higher-order statistical tests. Instead of applying the $Z$ tests immediately after the beginning of a diversion, they were applied after seven points under the assumption that an anomaly detection code would require seven points before the transient was discovered.

\section{Results of Analysis}

It was found that process monitoring alone offered little increase in MBP because of the natural PND and FAR of the system under the constraints suggested. However, Safeguards Envelope operation allowed for a 26\% increase in MBP. A table showing the PND and FAR of the PM systems for normal and Safeguards Envelope operation as 
well as their thresholds is found as Table B-1 of Appendix B. Figures 6 and 7 below show examples fifty diversion tests using these optimized thresholds for normal and Safeguards Envelope operation.

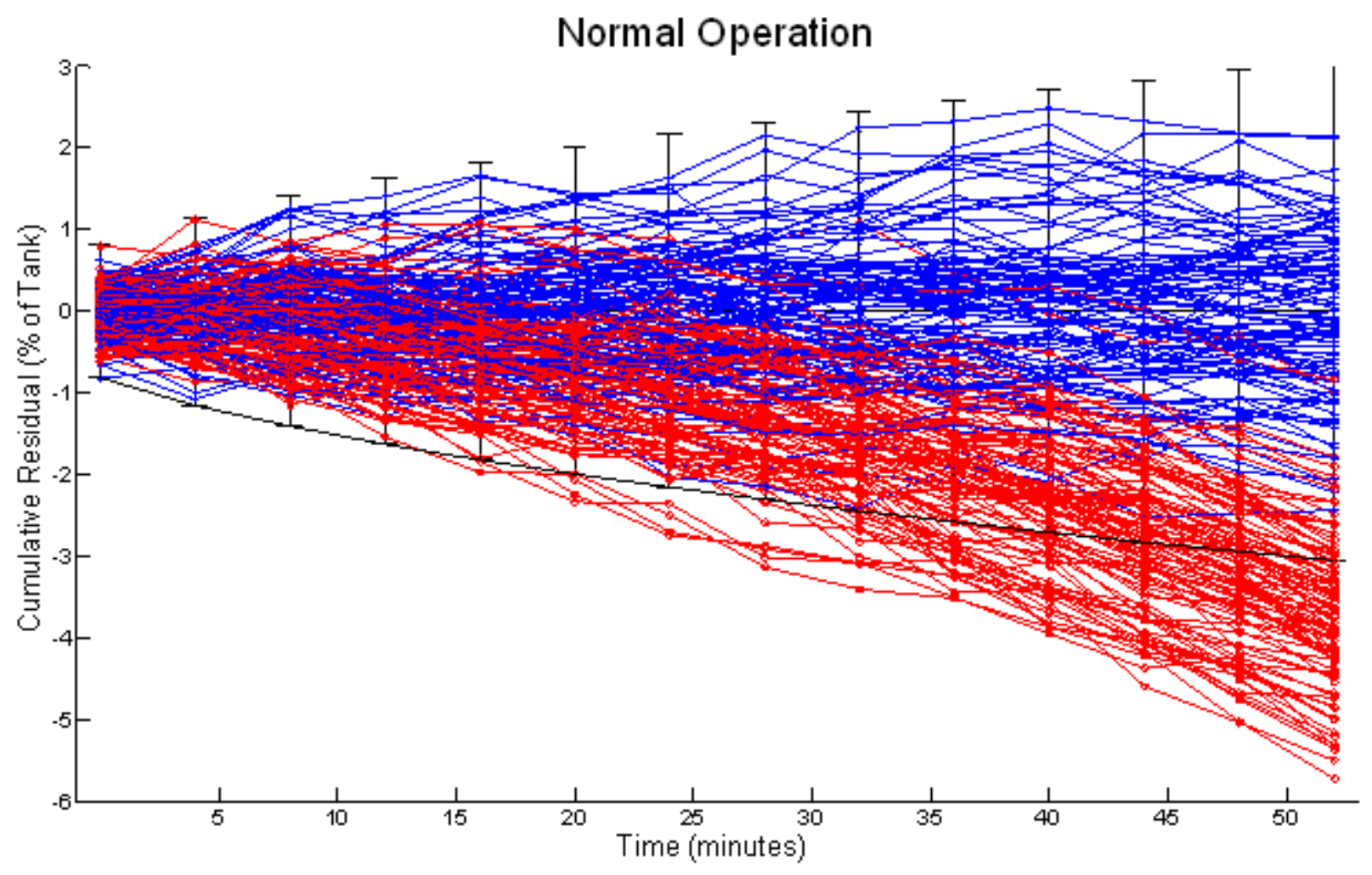

Figure 6. Little confidence has been gained using PM in addition to accountancy. 


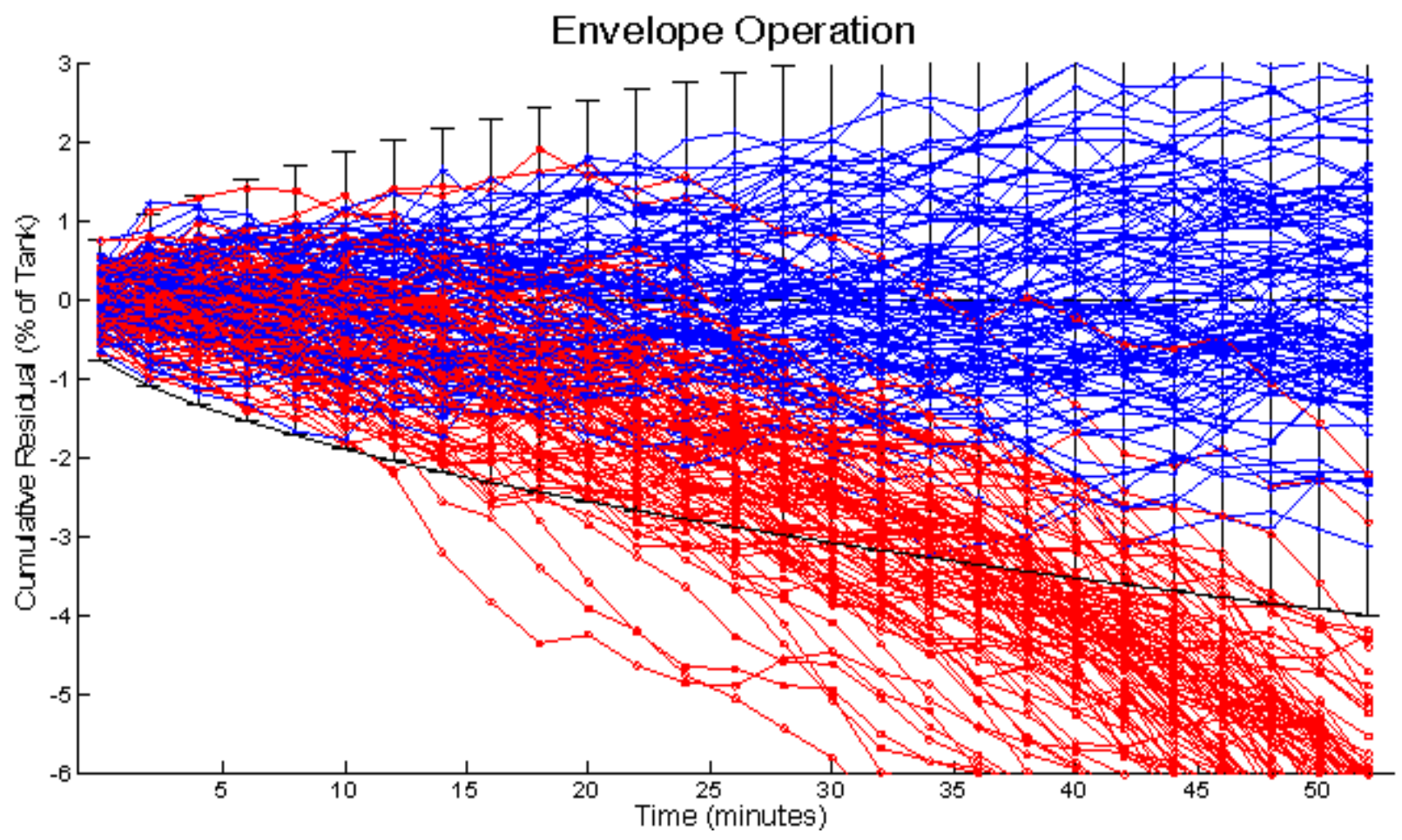

Figure 7. Diversions are more easily detected using Safeguards Envelope operation.

This $26 \%$ increase in MBP would have little value and relevance in modern facilities using NRTA but this MBP extension can be easily translated into additional confidence. It is possible that by switching to the second method intersection model, a drastic reduction in false alarm rates can be achieved with a minimal burden on increased accountancy.

In either case, reduction in standard MC\&A FAR must occur. Any system which has a false alarm rate greater than zero would currently cause the overall FAR to increase since accountancy is stated to be (and rightly so for efficiency) at the limit of the requirement. If MC\&A is exceeding requirements, there is some room to include process monitoring without altering current practices.

This example Safeguards Envelope was demonstrated over one attribute of one tank with only a single requirement but illustrates the concept of minor operational requirements to optimize detection of diversion. This concept is applicable to MC\&A practice adjustments, physical security concern inclusions, process monitoring as demonstrated, and future technologies as they come available.

\section{Analysis of Impact to Operations}

Operational impact is a required consideration for any new method or system. In the specific example described, the safeguards envelope required for half speed transfers 
in order to maximize detection by the process monitoring system. At first glance, it may appear that this requirement would enforce a reduction of $50 \%$ efficiency in the plant, an utterly unacceptable requirement. However, from observations of the data on the broader scope, this requirement does not appear to cause a significant burden to operations. Consider the following set of tank level data that surrounds Event \#1 in Figure 8.

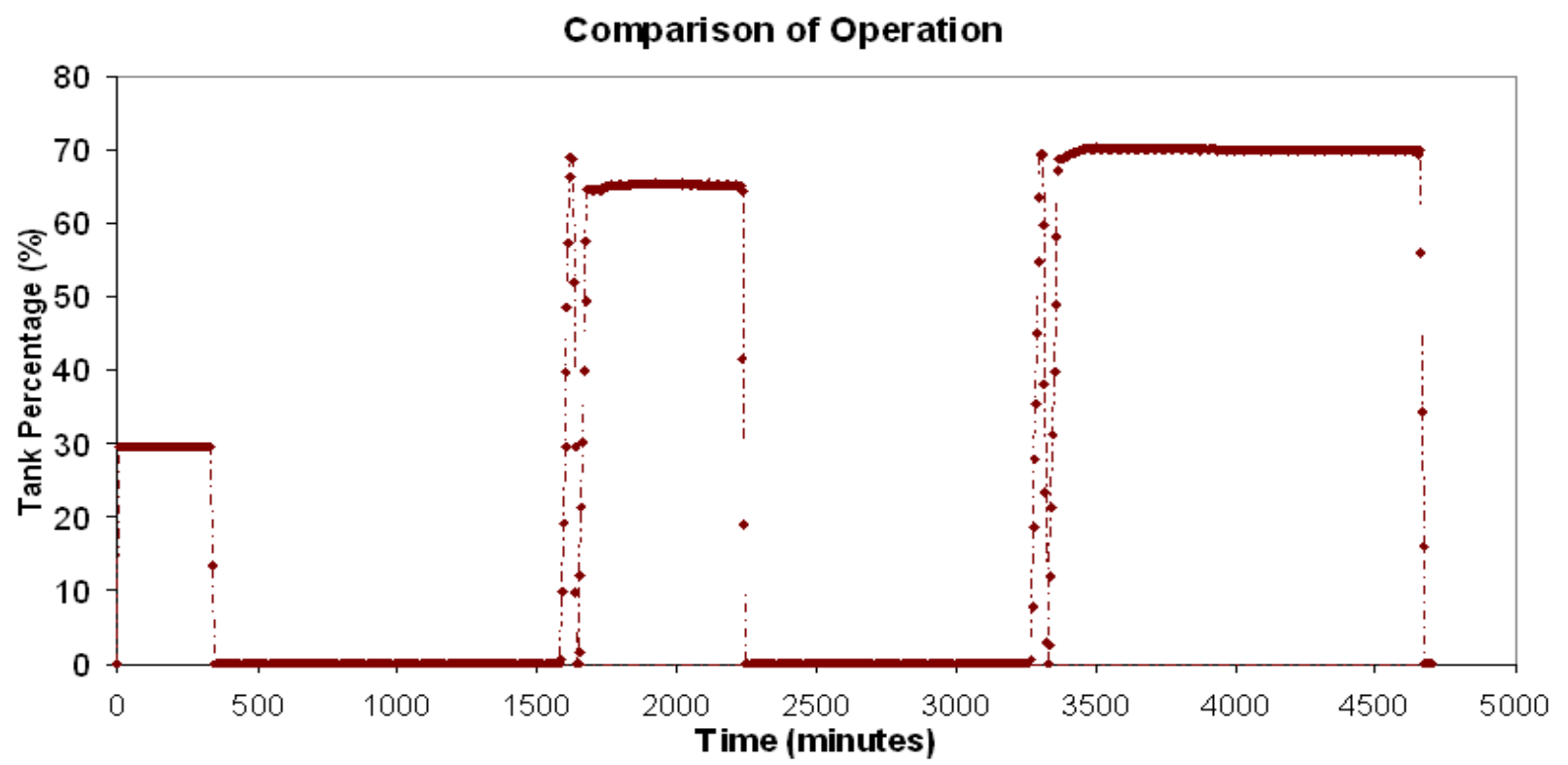

Figure 8. Event \#1 is a subset of this data set for a tank.

The tank spends slightly less than $2.5 \%$ of its time in a transient mode and even less time under fully transient conditions. As a result, under the assumption that transient conditions reduce the effectiveness of a tank's function by $50 \%$, the safeguards envelope described would reduce the efficiency of the plant by roughly $1.3 \%$. This could be significant but should be compared against the benefits of increased confidence, MBP, lowered FAR, and the ability to scale plants upwards. The Safeguards Envelope operation is shown below in Figure 9. Obviously a detailed analysis of the operational impact of a safeguards envelope is required, but a cursory analysis from the data indicates that the impact is expected to be minimal if requirements are designed properly. 


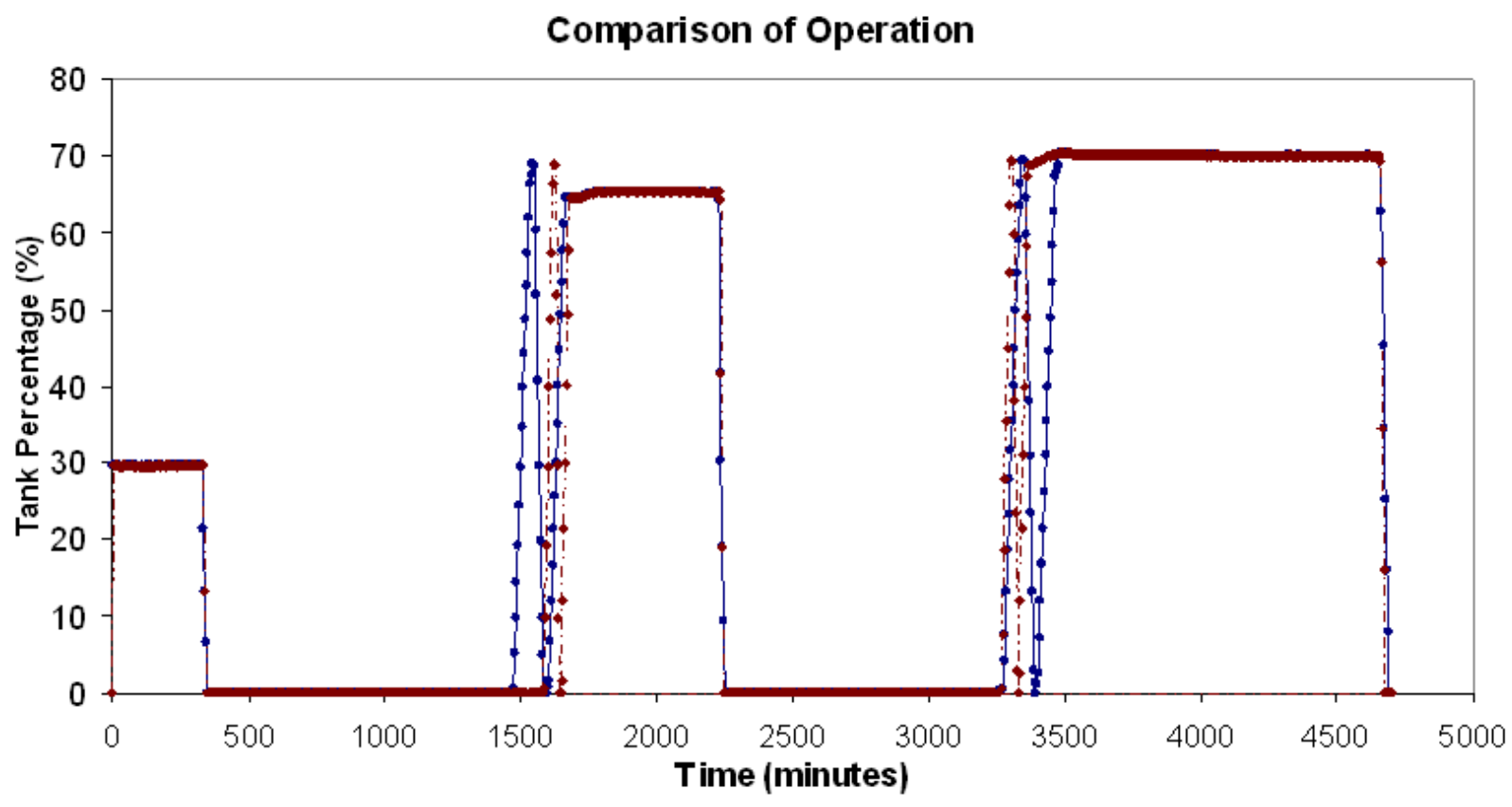

Figure 9. The Safeguards Envelope does not appear to significantly impact operations.

\section{EXPECTED CHALLENGES}

There are several challenges that are expected and considered, but not directly considered to be future work at this time. Ultimately, these challenges represent either constraints that must be considered carefully but cannot be eliminated or work that is sufficiently far in the future that they do not need to be directly addressed by the current research.

\section{Difficulty in Tight Requirements for Each Component}

If the Safeguards Envelope is applied in such a way that any of the many detection mechanisms is able to trigger an alarm (Union Model), each component must independently have a low FAR. This low FAR could push the PND higher rather than lower because of the strict requirements. In this case, any new addition to the Safeguards Envelope for consideration must be very well understood and applied technology. Any additional component with a significant FAR could actually hurt the overall system of safeguards. This can be understood intuitively by observation of Equations (1) and (2) of Appendix A. Equation (1) shows that FAR is linear under these assumptions while PND is multiplicative as shown in Equation (2). The approach for a Safeguards Envelope in which multiple systems must detect a diversion naturally creates a requirement for the PND of these systems to be low. If all systems must be triggered, the PND requirements may be prohibitive and there is a timeliness requirement to assure that all systems are synchronized. If any set of subsystems must show indication of a diversion, a balance may be struck, but this research is outside of the scope of the current and immediately considered projects. 


\section{Proprietary Data}

The Safeguards Envelope concept is very sound as a theory, but cannot be applied to maximum efficiency without a large data stream from the process observed. Data which lends itself to quick and easy determination of the exact path of nuclear material naturally leads to an easy determination of proprietary processes. As a result, a significant amount of research will be required in detection algorithms and ability to benchmark the algorithms with a minimal amount of confirmation. If these hurdles can be overcome, a "Green Light - Red Light" system may be devised in which the system only reports a binary status of "All Clear!" or "Requires Inspection." This would allow for the protection of proprietary information while still maintaining peak efficiency. ${ }^{12}$ These information barriers have been suggested for use in classified research in the United States as well as international safeguards approaches. ${ }^{13}{ }^{14}$ As information barrier methods evolve, the Safeguards Envelope method will become more and more valuable. The Safeguard Envelope methodology can be applied with any set of allowed systems, but it is possible that under severe data constraints, standard MC\&A practices may be the preferred verification system.

\section{Distinguishing Between Component Failure and Diversion}

All systems fail at some point and as additional complications are added to any system, the methods in which they can fail increase exponentially. Component failure of any part of a system could jeopardize the entire system without backup under the system in which any component can trigger an inspection requirement. As a result, the FAR may increase based on component failure of the subsystems which compromise the Safeguards Envelope. Furthermore, failure of non-Safeguards systems could increase FAR unless the failures of these systems can be simulated to high accuracy and the failures are included explicitly in the Safeguard Envelope creation. The cases presented in this paper are naturally ideal. Systems have been created in the past to determine component failure based on PM knowledge. Notably, the IGENPRO code suite (PRODIAG, PROTREN, and PROMANA) produced in 1997 used PM methods and fuzzy logic to determine failures before they actually occurred and an updated version of this or a similar system would help alleviate this problem. ${ }^{15}$

\section{FUTURE WORK}

While the expected challenges deal with issues of sufficient magnitude that they can only be mitigated, the future work described in the following pages can be accomplished in a reasonably short period of time. Future work includes the natural considerations of any project: scalability, applicability, expansion, and increases in efficiency. 


\section{Multi-Tank / Multi-Correlative Analysis for the Safeguard Envelope}

The current analysis is based on the observations of a single set of events for a single tank. Future research must expand to the other existing data for multi-tank and multiattribute correlations. Multi-tank correlations may be as simple as the statement that if Tank A empties into Tank B, when Tank $A$ empties there should be a corresponding increase in Tank $B$. They could, however, be more complex: maybe the pressure on a sample port in Tank B should move to a higher (known) pressure as Tank A fills Tank B because of temporary gas compression. Multi-attribute correlation analysis involves combining multiple measurements over a tank to determine the correct operations. In the case of the boiling of a tank, the density should increase and the tank level should decrease with the lost tank level equivalent to a density of unity. More complex correlations must be explored, especially in reference to expected diversion paths. As a stepping stone to this analysis, multi-variable, non-correlative analysis must be considered. This is discussed briefly in Appendix C. Future work will able to leverage this approach once the correlations between variables and tanks can be determined for different expected transients and diversion.

\section{Multi-Attribute Utility Analysis for Barriers to Proliferation}

Additional to the evaluation of the local possibility of diversion, a Multi-Attributes Utility Analysis (MAUA) may be used to locate the "weakest link" based on transient scenarios. While it may generally be accepted that certain areas of a facility have fewer barriers to proliferation than others, this "rule of thumb" may be false when the plant is operating outside of steady state. These expected transients, component failures, or possible diversions must be taken into account when applying safeguards by design or creating a safeguards envelope. One method to always maintain an understanding of the "weakest links" may be a MAUA method.

MAUA is a business decision analysis tool developed in $1978 .{ }^{16}$ It has several methods of compiling both quantitative and qualitative factors into a single decision based around the relative sizes of single figures of merit. Each factor is associated with some hurdle or aspect of a decision that must be taken into account. In safeguards, when evaluating proliferation resistance (PR), this typically refers to different levels of risk, time, money, or effort in order to successfully divert material. This can be accommodated in a MAUA analysis to assess the relative PR among various points. These factors are assessed by the creation of a utility function for each factor by the analyst. These utility functions generate a specific value based on their input parameters. These specific values are then weighted and compared to a standard or set of standards. In safeguards, these would represent on-normal, off-normal, and expected diversion path standards. MAUA methods have been applied successfully to evaluate the PR of SNM as a function of time and location in the nuclear fuel cycle with results that were consistent with expert elicitation. ${ }^{17}{ }^{18} \mathrm{~A}$ detailed and mathematical description of the MAUA methodology can be found in Appendix $D$ and the specific barriers to proliferation approach to a safeguards envelope can be found in a previous paper. ${ }^{19}$ 
When designing a facility or creating a safeguards envelope, the most basic safeguards envelope would be the operational parameters which require that operations not take actions which lower the PR below a certain threshold. This may help indicate additional containment and surveillance, heightened security, or attention to detail required during certain transients.

\section{Multi-Attribute Utility Analysis for Diversion Detection}

It is also possible to perform the diversion detection test using MAUA methods. In this case, several diversions would be simulated with varied weighting structures and utility analyses in a Markov Monte Carlo method. It is recommended for computational efficiency that the utility functions remain constant for each diversion type, but the optimized weights for each "type" of diversion tested against would be different. In this scenario, data that is collected would be input to the MAUA for utility function evaluation, and then a series of weights and optimized threshold would be applied for each diversion to minimize PND and FAR. There are significant merits and flaws to this approach: the method would be fast and possibly detect diversions at a higher rate than generic algorithms but requires an understanding of which diversion paths are most significant and one of the assumptions of the MAUA method (attributes are independent) could be violated. It is likely that this method will be investigated in the future but not given full attention as it appears that other venues will yield more relevant results.

\section{Higher Level Statistics Analysis}

The current anomaly detection method is the rudimentary $Z$ probability test. Hundreds if not thousands of more advanced anomaly detection methods exist. Several of these have been explored, but not applied to nuclear transient scenarios. ${ }^{20}$ Fuzzy logic, kernel regression methods, principle component analysis, sequential hypothesis testing, and fractal analysis (for the semi-analytic method) are all possibilities for future anomaly detection. These methods lend themselves well to computer-based optimization and have already been verified by use in other fields.

\section{Simulation Work}

As the theory of the safeguards envelope is demonstrated conclusively, it is clear that future applications will require integration into new simulation software. By running optimization schemes (possibly automated, see below), suggested operating envelopes can be created for facilities that only exist ethereally. This adds a new dimension of analysis to consider in an automated format.

\section{Neural Network/Automated Optimization}

With the application of higher-level statistics analysis and multi-dimensional correlations, normal exploration of the parameter space becomes computationally 
expensive and inefficient. Because of this expense, true optimums are unlikely to be found without the use of automated optimization. Neural network learning methods appear the natural choice, but other optimization methods, such as Markov point methods combined with Bayesian probabilities, will be considered. It is likely that the highest benefit of the safeguards envelope will be realized based on correlations which are not intuitively obvious, hence the explicit requirement for automated optimization.

\section{Scalability Studies}

The ICPP data is the most complete data set available currently in the DOE complex, but the ICPP was not of the scale of current and expected future reprocessing complexes. Standard MC\&A practices, such as NRTA, only show limitation during scaling up of the plant. The Tokai Reprocessing Facility allows significantly more time between MBPs compared to the Rokkasho Reprocessing Plant because of scaling. In the same way, before the Safeguards Envelope methodology can be considered appropriate for future reprocessing facilities, scalability studies must be performed. These scalability studies should confirm that the addition of the Safeguard Envelope methodology will increase the confidence of nuclear material. Because the majority of the ICPP data was reported in percentage, it is likely that these studies will proceed rapidly. There are some factors which do not scale linearly, however, and these will be explicitly addressed.

\section{Operational Impact Studies}

The current analysis has expressed explicitly; there does not appear to be a significant impact to operations by the application of the Safeguard Envelope requirements. However, this statement was extrapolated from the data alone and not operator experience. A detailed analysis of the operational impact of a multi-attribute based Safeguard Envelope is required. No system should be researched which does not have the ability to actually be applied effectively, so future work will include such an analysis. It is unlikely that an economic efficiency can be determined because the actual amount of resources required by safeguards for existing plants is very likely not available in the public literature. However, estimates will be made based on open source information, will likely be within a standard deviation of true efficiency, and should set a baseline for future detailed analyses. Any operating plant should have adequate models for the cost of safeguards measurements and operational changes. Our goal is to develop the impact of operational changes on safeguards effectiveness to aid the plant in finding the most cost-effective balance. 


\section{APPENDIX A \\ Detailed Analysis of Process Monitoring Benefit}

\section{Theory}

The usefulness of PM data depends on its effect on the overall sensitivity and specificity of the monitoring system. Assuming probabilities of causing an alarm are independent, we can reason for the union model that

$\alpha=\alpha_{A}+\alpha_{P M}-\alpha_{A} \alpha_{P M}$,

where

$\alpha=$ the overall rate of safeguards false alarms

$\alpha_{A}=$ the rate of false alarms due to accountancy safeguards

$\alpha_{P M}=$ the rate of false alarms due to process monitoring safeguards

and that

$\beta=\beta_{\mathrm{A}} \beta_{\mathrm{PM}}$

where

$\beta=$ the overall rate of failure to detect a diversion of one significant quantity

$\beta_{A}=$ the rate of failure due to accountancy measures failing to detect such a diversion

$\beta_{P M}=$ the rate of failure due to process monitoring measures to detect such a diversion.

As expected, in the case where no PM system is used $\alpha_{P M}=0$ and $\beta_{P M}=1$ implying $\alpha_{0}$ $=\alpha_{A}$ and $\beta_{0}=\beta_{A}$. In the intersection model, both systems' alarms are needed for an alert.

$\alpha=\alpha_{A} \alpha_{P M}$

$\beta=\beta_{A}+\beta_{P M}-\beta_{A} \beta_{P M}$

One must note that Equation (4) implies that in the intersection model both sensitivities must be much smaller than a desired $\beta_{0}$. The only flexibility this model affords is the sharp decrease in FAR, which allows alarm thresholds to be set much lower. This is questionable in practice, since such high confidence in PM detection may be unfounded. 


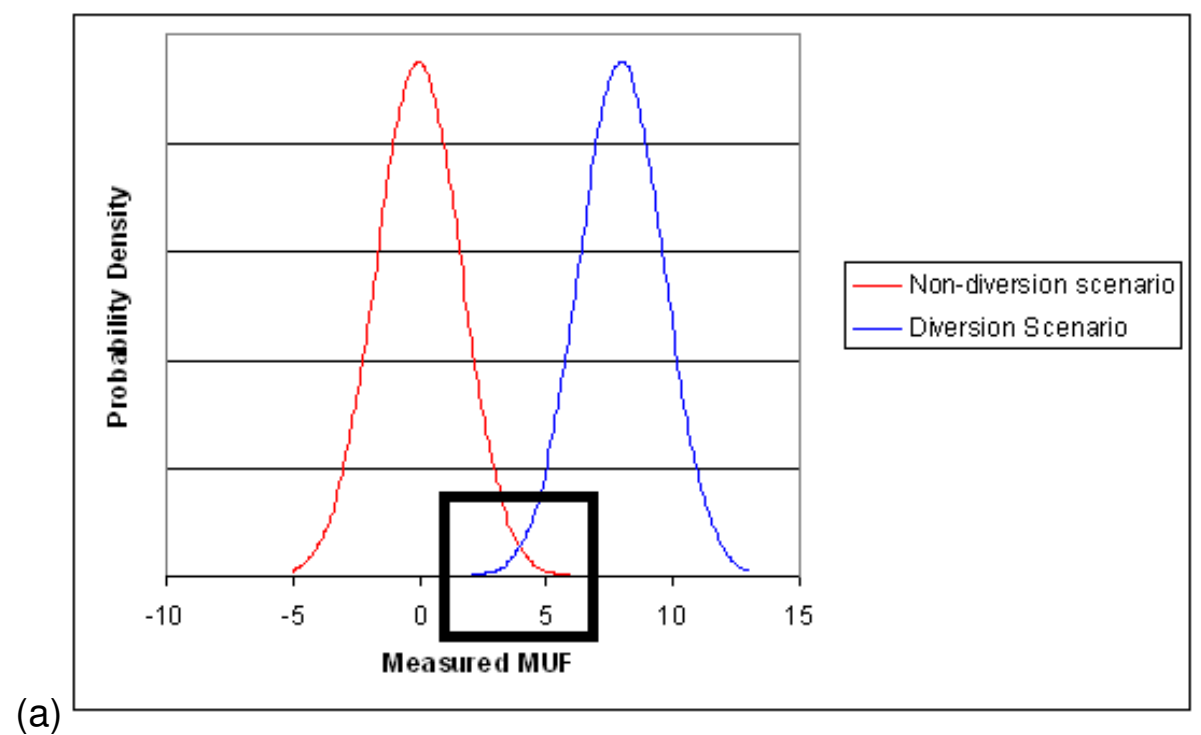

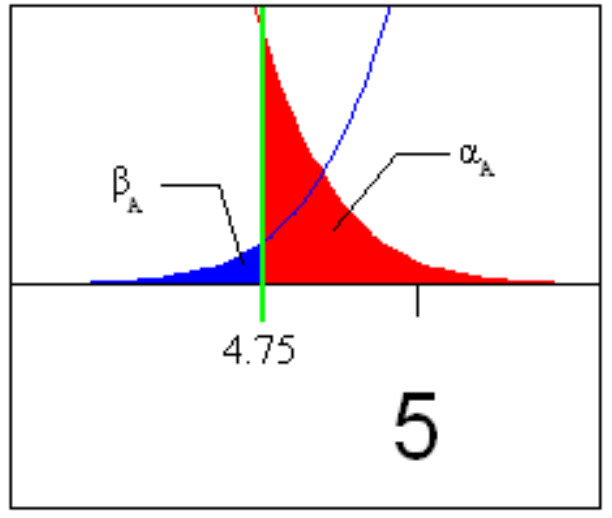

(b)

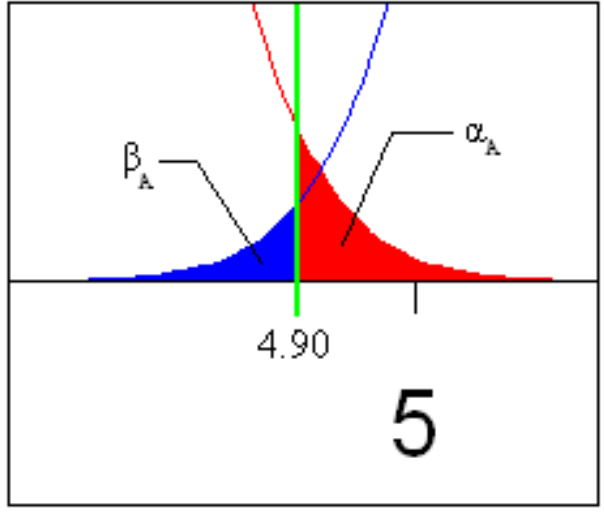

(c)

Figures $A-1 a, A-1 b$, and A-1c. Expected data distribution of normal and divergent MUF measured; areas showing PND and FAR for material unaccounted for alarm threshold (MUFAT) are of a) 4.75 and b) $4.90 \mathrm{~kg}$. These exemplify the dependence of $\beta_{\mathrm{A}}$ and $\alpha_{\mathrm{A}}$ on the MUFAT.

One may estimate the maximum $\sigma_{A}$, the standard deviation of the accountancy system, given a specific $\alpha_{A}$ and $\beta_{A}$ by Equation (5).

$\sigma_{\mathrm{A}} \leq 1 \mathrm{SQ} /\left[\right.$ inverseNorm $\left(\alpha_{\mathrm{A}}\right)$ - inverseNorm $\left.\left(\beta_{\mathrm{A}}\right)\right]$

where

$\sigma_{\mathrm{A}}=$ the acceptable standard deviation in accountancy measurements $S Q=$ a significant quantity of nuclear material 
where the inverseNorm $(p)$ function is defined as the point where a cumulative Gaussian distribution reaches $p$.

If it is assumed that the standard deviation increased linearly with MBP:

$\mathrm{MBP}=\mathrm{MBP}_{0} \sigma_{\mathrm{A}} / \sigma_{\mathrm{A}, 0}$

where

$\mathrm{MBP}_{0}=$ the MBP of current accountancy measurements

$\sigma_{\mathrm{A}, 0}=$ the standard deviation of the same.

\section{Results}

This analysis estimated $\sigma_{\mathrm{A}, 0}$ as $1.69 \mathrm{~kg}$ using Equation (5) and assuming current tests achieve exactly $0.1 \%$ PND at a 5\% FAR over 8-day MBPs. Using Equations (1-4), the required $\alpha_{P M}$ and $\beta_{P M}$ needed to reproduce the same overall detection sensitivity and specificity with the additional confidence of PM oversight were estimated and are shown in Figure 2.

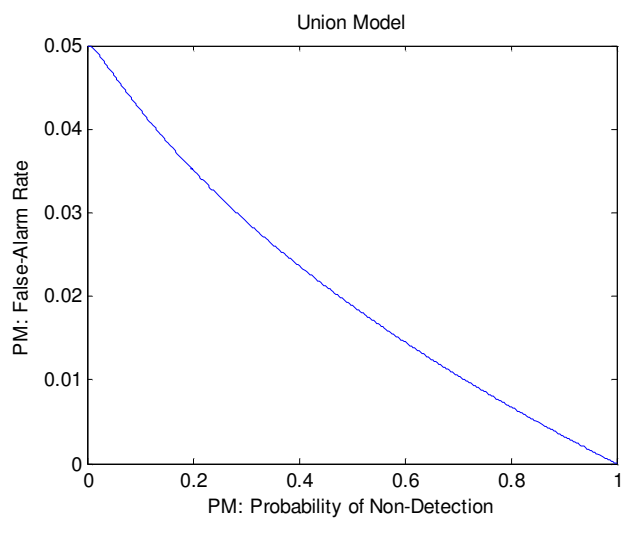

(a)

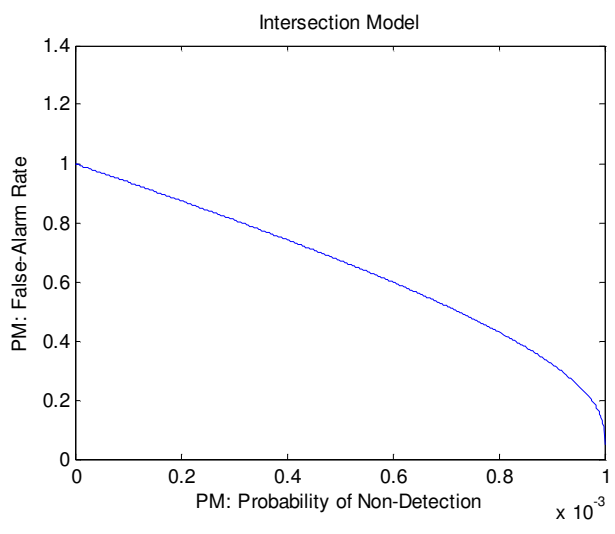

(b)

Figures $A-2 a$ and $A-2 b$. Threshold of usefulness for $P M$ in a) union and $b$ ) intersection analysis. Methods that fall above the curve produce too many false alarms to be useful. Note plots' different scales.

Using Equation (5), estimation was performed of the standard deviation of accountancy measurements which will allow sufficient accuracy to meet monitoring standards with PM (Figure 3), and by using Equation (6), approximate the length of a material balance period now allowable as shown in Figure 4. 


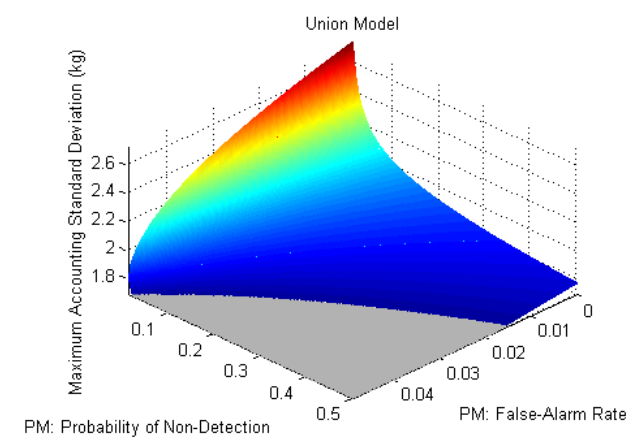

(a)

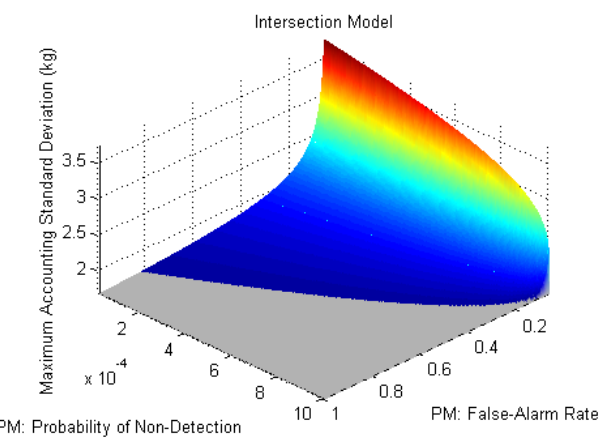

(b)

Figures A-3a and A-3b. Maximum allowable standard deviation of accountancy data for given PM characteristics. Note that the (lower) gray surface represents a region below the threshold of benefit.

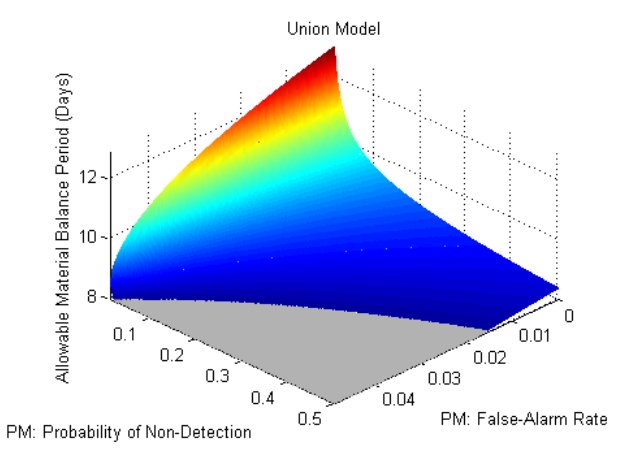

(a)

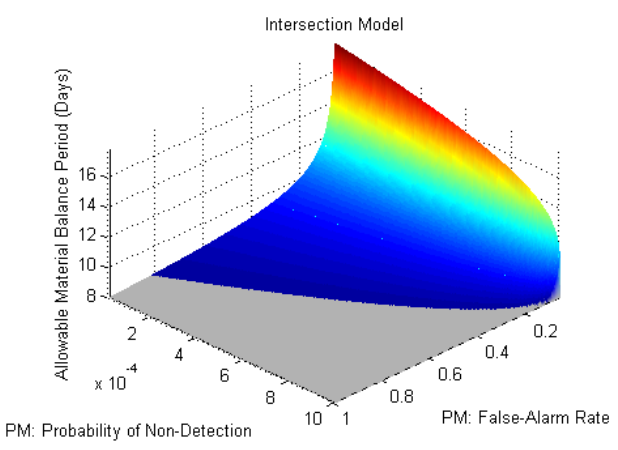

(b)

Figures A-4a and A-4b. Maximum allowable MBP for given PM characteristics. Note that the (lower) gray surface represents a region below the threshold of benefit.

The allowable MBP grows without bound as $\beta_{\mathrm{PM}}$ approaches $0.01 \%$ for the Union model or $\alpha$ approaches $5 \%$ for the Intersection model. Of course, if one can reach an acceptable level of confidence strictly by PM, accounting becomes unnecessary. It is not this paper's contention that only using PM safeguards are cost effective or even possible; the method contained simply represents a function to test the usefulness of PM analysis or analyze the most effective way to set PM thresholds to reduce the burden on accounting measurements. 


\section{APPENDIX B \\ Simulation and Optimization of Process Monitoring Safeguards by Semi-Analytical Methods}

To approximate data made available by individual PM instruments, one uses a Gaussian distribution. For the suggested method of unifying multiple data sets (the Malahalanobis distance ${ }^{21} 22$ ), the data is expected to fall into a $x^{2}$-distribution.

Our favored method for anomaly detection is a series of successive Z-tests; first on the residuals (variation from an expected value) of the first measurement, then on the sum of the residuals from the first two measurements, etc. This analysis of residuals makes it very difficult to perform a purely analytical calculation of false alarm rate and the probability of non-detection of material diversion at a given alarm threshold.

\section{Theory}

Using a normal distribution of data (or $\mathrm{X}^{2}$-distribution for multivariate data), we estimate the fraction of readings that fall into a set of ranges. We then zero-out the fraction that would trigger an alarm and then redistribute the remaining fractions according to the distribution of the next measurement to be taken (see Figures. B-1 and B-2). After repeating for every time measurement to be taken, we sum the region to find the fraction of cases which would not produce an alarm.

The alarm threshold changes as the number of measurements increases. A representative parameter, the alarm probability, represents the probability that diversion-free operation will produce an alarm in any give measurement. The alarm threshold widens as the square-root of the number of measurements taken.

We can use the above procedure to find the FAR and PND at a given PM alarm probability. Then using analytical statistics to estimate the allowable MBP at a given PM alarm probability as shown in Appendix A, we found the threshold which allowed the longest MBP.

The entire analysis was compared to a half-speed tank transfer, which would record twice as many (28) data points. 


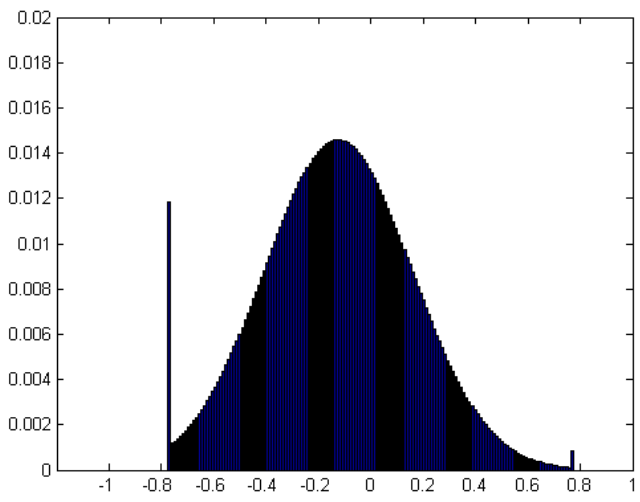

(a)

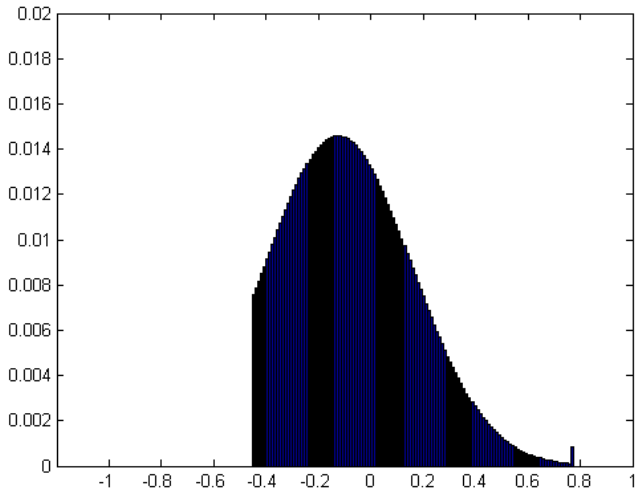

(b)

Figures B-1a and B-1b. A Gaussian distribution of measurement residuals is discretized. b) Measurements below the alarm threshold are removed, (i.e., set to 0).

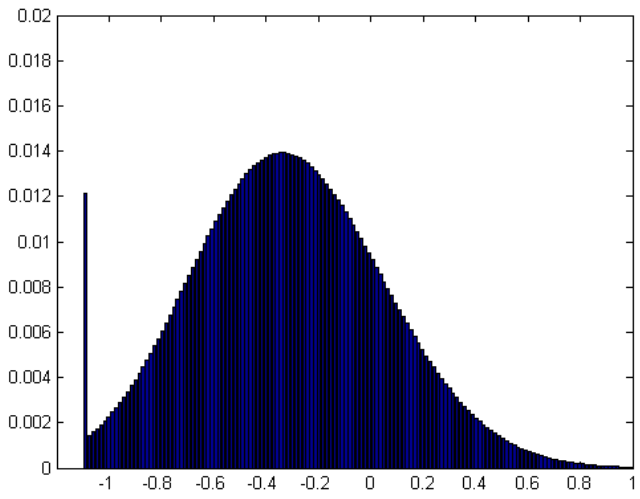

(a)

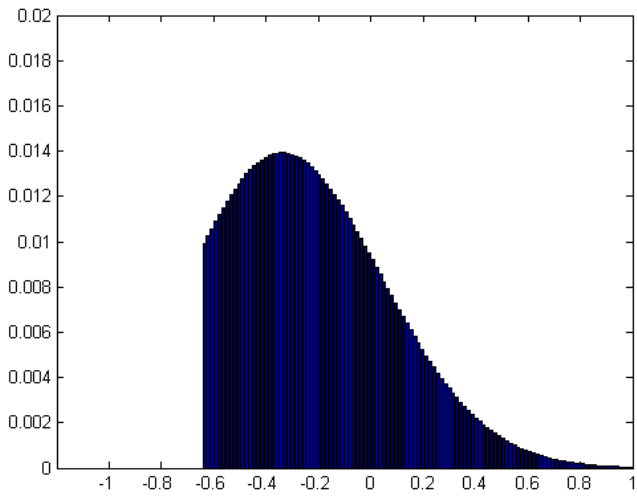

(b)

Figures $\mathrm{B}-2 \mathrm{a}$ and $\mathrm{B}-2 \mathrm{~b}$. .. a) Each mesh is redistributed into another normal distribution. The sum of these normal distributions produces a skewed distribution approximating the cumulative residuals of cases where the first measurement did not produce an alarm. b) The threshold is adjusted to account for spreading expected in the distribution.

Overall, we expect stark improvement in detection as the number of taken measurements increases. The Markov simulations in Figure B-3 demonstrate a parabolic change in the cumulative difference. As more measurements are taken, the cumulative residual increases quadratically. 


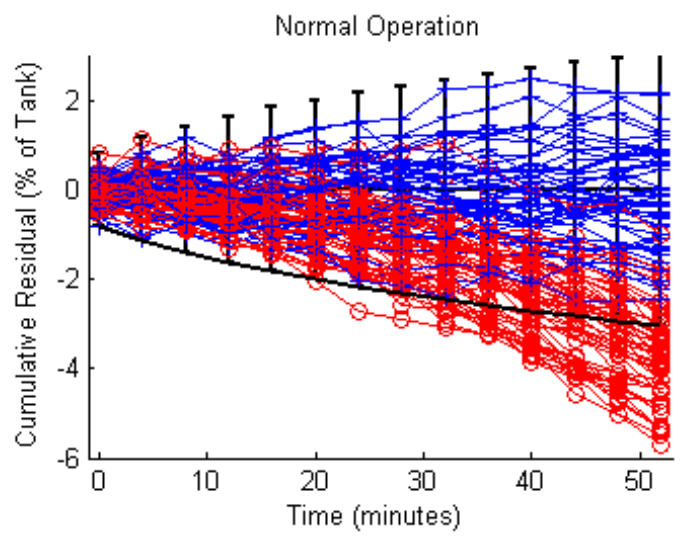

(a)

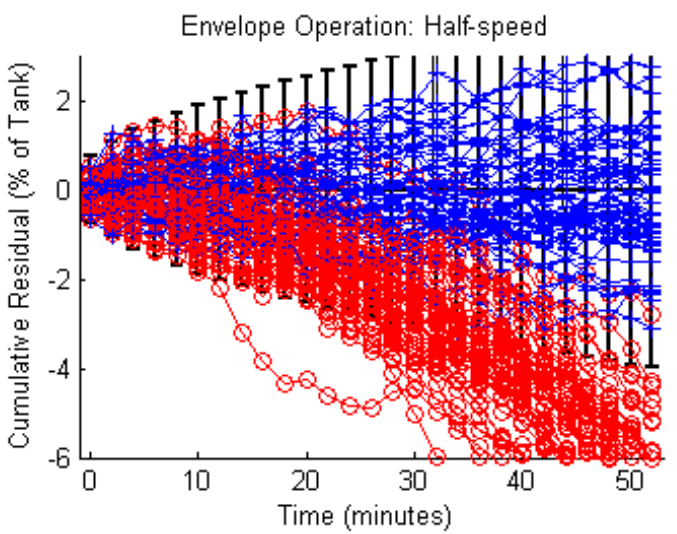

(b)

Figure. B-3a and B-3b. The parabolic drop in cumulative residual occurs much more quickly when more measurements are taken (b) than during normal operation (a); thus diversions (shown in red) are much easier to distinguish from normal operation (blue).

\section{Results}

Data from the Idaho Chemical Processing Plant indicates that approximately 14 data points are recorded during the short transients. The maximum allowable MBPs are listed in Table B-1.

Table B-1. Comparison of findings for normal operation and operations at half-speed.

\begin{tabular}{|c|c|c|c|c|c|c|}
\hline Speed & $\begin{array}{c}\text { Measure- } \\
\text { ments }\end{array}$ & $\begin{array}{c}\text { Optimized PM } \\
\text { Alarm Probability }\end{array}$ & $\begin{array}{c}\text { Total } \\
\text { Diversion }\end{array}$ & FAR & PND & $\begin{array}{c}\text { Allowable } \\
\text { MBP (days) }\end{array}$ \\
\hline Full & 14 & $1.9610 \mathrm{e}-3$ & $-0.5 \%$ & $1.39 \%$ & $23.2 \%$ & 8.57 \\
\hline $\begin{array}{c}\text { Half- } \\
\text { speed }\end{array}$ & 28 & $3.3295 \mathrm{e}-3$ & $-0.5 \%$ & $2.91 \%$ & $1.41 \%$ & 10.85 \\
\hline
\end{tabular}

\section{Conclusion}

This limited one-variable demonstration does not fully simulate the complexity of an entire facility's measurements. It does show that operational parameter changes can have significant impact on the sensitivity of safeguards systems. As more variables are considered, however, the semi-analytic method may become less valuable because each dimension will add an exponent to the computational cost. 


\section{APPENDIX C Early Multi-Correlative Analysis}

\subsubsection{Foundation of Theory}

For analysis of multiple variables, a series of Markov simulations analyzes the alarm rate during possible diversions more effectively than purely analytical codes. Here we use random numbers to simulate signal noise, then shift the measurement to simulate a diversion event. We then determine whether the diversion can be distinguished from normal operation.

Markov simulations involve producing many representative examples and looking at the overall trend they produce. Markov methods are favored over semi-analytical methods for more complex systems because of the complexity of categorizing multiple variables. Memory requirements for reasonable fidelity would grow exponentially when meshing multivariate systems.

Additionally, Markov methods allow for simpler optimization because data from each run can be compactly stored. Once a process is fully simulated, our code only records the threshold which would trigger an alarm.

\subsubsection{Theory}

Assuming accurate predictive models are developed, the detection of a diversion of a special quantity (SQ) of SNM depends on the ratio of the amount of change a diversion represents in PM measurements to the standard deviation of those measurements from the true value. If the standard deviation is too high, the diversion will be masked by noise.

To examine this ratio, we simulate normal operating conditions and protracted diversions from appropriately sized tanks at the beginning and end of a reprocessing facility (see Table C-1.) All ratios assume a standard deviation of $0.2 \%$ of the maximum or expected value, the appropriate IAEA target value.

Our simulation generates random normally-distributed numbers to represent measured values, then changes the data to reflect a diversion. Each type of diversion has a characteristic signature: a graduated increase to a maximum amount (listed as "change/standard deviation" in Table I) which is either sustained (as in diversion I) or gradually decaying away (as in diversions II through IV). This reflects the fact that the material stolen would remain missing if it is not replaced, while a density change would fade away as material flows into and is drawn from the tank. Our code picks a random number of measurements over which the diversion occurs, a random starting place, and in diversions II through IV the diversion change decays to half of its peak value at the end of the measurement period (see Figure C-1).

The script then ran the simulated data against several tests which appear reasonable for the generic detection of nuclear material theft: $a x^{2}$-test over all variables on the last run, a Z-test over each variable on the last measurement, the last 10 measurements, the last 20 measurements, all measurements taken at the time, and all measurements taken overall. 
Table C-1. Diversion profiles used in Markov simulations.

\begin{tabular}{|c|c|c|c|c|c|}
\hline & $\begin{array}{l}\text { No } \\
\text { diversion }\end{array}$ & Diversion I & Diversion II & Diversion III & Diversion IV \\
\hline & & $\begin{array}{l}\text { Removal } \\
\text { without } \\
\text { replacement }\end{array}$ & \begin{tabular}{ll}
\multicolumn{2}{l}{ Replacement } \\
with HM nitric \\
acid $\quad$ (front \\
end) &
\end{tabular} & $\begin{array}{l}\text { Replacement with } \\
\text { pure nitric acid } \\
\text { (front end) }\end{array}$ & $\begin{array}{l}\text { Replacement } \\
\text { with pure nitric } \\
\text { acid (back end) }\end{array}$ \\
\hline Tank volume (L) & Arbitrary & 16000 & 20000 & 20000 & 1000 \\
\hline $\begin{array}{l}\text { Change in } \\
\text { measurement }\end{array}$ & 0 & $80 \mathrm{~L}$ & $\begin{array}{lr}-1 \quad \mathrm{~g} / \mathrm{L} & \text { for } \\
\text { fraction } & \text { of } \\
\text { tank } & \text { stolen } \\
(40 \%) & \\
\end{array}$ & $\begin{array}{lrr}-271 \quad \mathrm{~g} / \mathrm{L} & \text { for } \\
\text { fraction of } & \text { tank } \\
\text { stolen }(40 \%) & \end{array}$ & $\begin{array}{l}250 \quad \mathrm{~g} / \mathrm{L} \text { for } \\
\text { fraction of tank } \\
\text { stolen }(3.2 \%)\end{array}$ \\
\hline $\begin{array}{l}\begin{array}{l}\text { Density } \\
(\mathrm{g} / \mathrm{L})\end{array} \\
\text { of } \mathrm{Pu}\end{array}$ & arbitrary & 100 & ( & 1 & 250 \\
\hline $\begin{array}{l}\text { Volume change / } \\
\text { standard } \\
\text { deviation }\end{array}$ & 0 & -2.5 & 0 & 0 & 0 \\
\hline $\begin{array}{l}\text { Density change / } \\
\text { standard } \\
\text { deviation }\end{array}$ & 0 & 0 & -0.16 & -43.36 & -3.2 \\
\hline $\begin{array}{l}\text { Temp. change / } \\
\text { standard } \\
\text { deviation }\end{array}$ & 0 & 0 & 0 & 0 & 0 \\
\hline
\end{tabular}

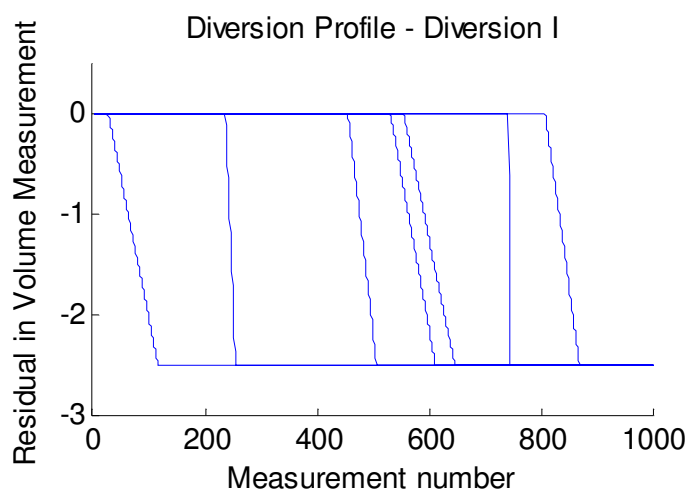

(a)

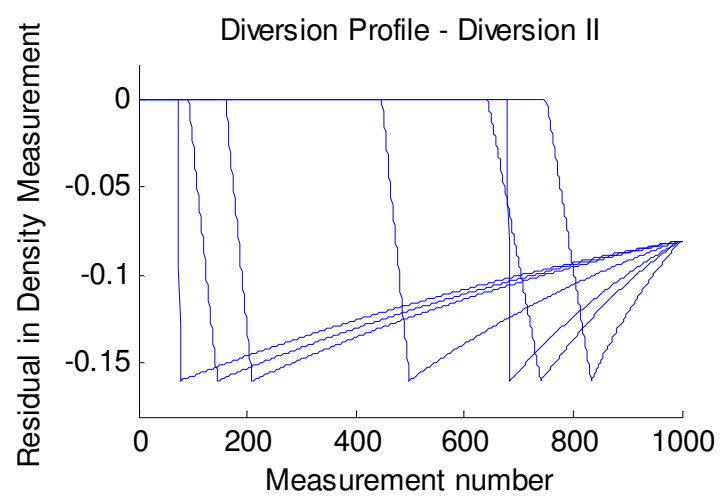

(b)

Figures C-1a and C-1b. Example diversion profiles for diversion type I (a) and diversion type II to exemplify diversion types II through IV (b).

Each of these tests returns a probability - the probability of achieving data this variant under normal operations - which is recorded. An appropriate probability threshold to signal alarm was determined after the fact; in plant operations, these must be estimated before operation based on simulated data. 
The test ran (1) as many Markov simulations as necessary to achieve a desired confidence (1.4\%) at an assumed probability threshold or (2) 100 simulations, whichever was larger.

\subsubsection{Results}

As Table C-2 shows, at a reasonable rate of false alarms, only the most unlikely diversion scenario was frequently unnoticed.

Table C-2. Alarm rates for different detection scenarios. Standard deviation of the alarm rates is $\sim 1.4 \%$.

\begin{tabular}{|l|l|l|l|l|l|}
\hline & $\begin{array}{l}\text { No } \\
\text { Diversion }\end{array}$ & Diversion I & Diversion II & $\begin{array}{l}\text { Diversion } \\
\text { III }\end{array}$ & $\begin{array}{l}\text { Diversion } \\
\text { IV }\end{array}$ \\
\hline $\begin{array}{l}\text { Number of } \\
\text { Markov } \\
\text { simulations }\end{array}$ & 275 & 100 & 1167 & 100 & 100 \\
\hline Alarm Rate & $5.82 \%$ & $100 \%$ & $37.02 \%$ & $100 \%$ & $100 \%$ \\
\hline
\end{tabular}

\subsubsection{Conclusion}

While all other diversions were obvious, PM data has some difficulty detecting removal and replacement with very well-simulated surrogate (e.g., Diverion II). This is indeed the most unlikely diversion scenario and the hardest to achieve; more accurate estimates of possible disruption in the PM measurements (such as temperature changes or small fluctuations in level) may show this scenario is very detectable. 


\section{APPENDIX D \\ Multi-Attribute Utility Analysis (MAUA) Theory}

Though each of the PR analyses has its merits and limits, the MAUA methodology's limits appear to be the most manageable and the specificity, updatability, and transparency of the MAUA analysis method makes it the preferred choice. The application of the MAUA methodology for the PR assessment of FBRFC fits well into the PR\&PP framework proposed by the Generation IV (GEN IV) International Forum (GIF). The GIF PR\&PP has identified threat scenarios for the PR assessment and has proposed six preliminary measures each for estimating the PR and PP of nuclear energy systems. The proposed measures for PR are (1) Proliferation Technical Difficulty, (2) Proliferation Resources, (3) Proliferation Time, (4) Fissile Material Quality, (5) Detection Time, and (6) Detection Resources.

MAUA is a well-established decision analysis methodology and has evolved since its first publication in 1978. ${ }^{23}$ The MAUA method has been applied, for example, in air defense ${ }^{24}$, bridge construction and strengthening ${ }^{25}$, electronic commerce architecture ${ }^{26}$, and house value evaluation ${ }^{27}$. In order for analysts to maximize their benefit, objective functions were created that can ultimately compare competing values.

MAUA consists of several methods of compiling multiple factors in order to make a single decision. Because there are several methods, MAUA analysis ranges from simple weighted addition and multiplication to algorithms of sufficient complexity that high end computing is required. Attribute values aid in ascertaining conclusions regarding the possibility of material diversion at a given rate under certain conditions of risk. These attributes are used in composite or weighted form to make a final assessment. This final, weighted form is the ultimate goal of any MAUA method, an objective function, commonly referred to as the utility function, that consists of multiple attributes which best describe the attractiveness of a system. The best decision, obviously, is one which maximizes the value of the function.

The various steps involved in the MAUA assessment are:

- Define utility function $u\left(x_{1}, x_{2}, \ldots . . x_{i}\right)$ to represent value of a given path for a range of attribute values $x_{i}$

- Define the single-attribute utility functions $u_{i}\left(x_{i}\right)$ that describe this overall utility

- Define a set of attributes, $\left\{x_{i}\right\}$, that can be related to cost, time, material quality, or other characteristics deemed of value or utility

- Apply these attributes and utility functions into a MAUA weighting scheme.

Differences between MAUA analyses include the use of different weighting schemes, the use of various utility functions, and the selection of different attributes for inclusion. ${ }^{28}$ Three equations are shown to illustrate the differences between potential methods. In all equations, $u$ is the benefit (utility) that we wish to maximize, the $k$ values are weights determined by the analyst, $u_{i}$ is a utility function for a single attribute, the $x$ values are attributes which feed into the utility functions and the $k$ value is determined by the normalization of the function (this allows the $k$ values to be undounded). Pure additive 
MAUA (Equation [1]) is limited because as the utility of an attribute goes to zero there may be little impact in the final result. Adding factors with low sensitivity will tend to make the entire process less sensitive and subsequently give less information. Purely multiplicative weighting scheme (Equation [2]) is limited in that the addition of attributes naturally causes the function to decrease (as any new utility function naturally will be less than unity). Very complex algorithms are computationally expensive, difficult to explain to policy-makers, and generally formed for specific applications. As a result, an adaptation of the multiplicative MAUA has been chosen (Equation [3]).

$u\left(x_{1}, x_{2} \ldots x_{i}\right)=\sum_{1}^{n} k_{i} u_{i}\left(x_{i}\right)$ (Pure additive form)

$u\left(x_{1}, x_{2} \ldots x_{i}\right)=\prod_{i}^{n} k_{i} u_{i}\left(x_{i}\right)$ (Pure multiplicative form)

$1+K u\left(x_{1}, x_{2} \ldots x_{i}\right)=\prod_{i}^{n}\left(1+K k_{i} u_{i}\left(x_{i}\right)\right)$ (Appropriate Multiplicative Form)

In order to apply our preferred multiplicative form, there are several additional requirements. First, the sum of all the weighting factors must not be exactly 1.0, because that would reduce the equation to the additive utility function. So the sum of all weighting factors will be selected as greater than 1. Also, the weighting factors cannot each be equal to 1.0. If they were, the only solutions to Equation (4) would be $K=-1$ and $K=0$, neither of which are valid. The solution must lie on $-1<K<0$ when $\sum_{1}^{n} k_{i}>1$ (the multi-attribute utility function exhibits risk aversion). If $\sum_{1}^{n} k_{i}<1$, the multi-attribute utility function exhibits risk seeking, and hence will not be used. The solution to find $K$ in the range $-1<K<0$ for the normalizing condition in Equation (3) for a typical case is demonstrated in Figure $D-1$. In this case, 10 attributes $x_{i}$ of equal weights $k_{i}(i=1,10)$ with $\sum_{1}^{n} k_{i}=2$ are assumed for the utility function representing a particular path in the system. 


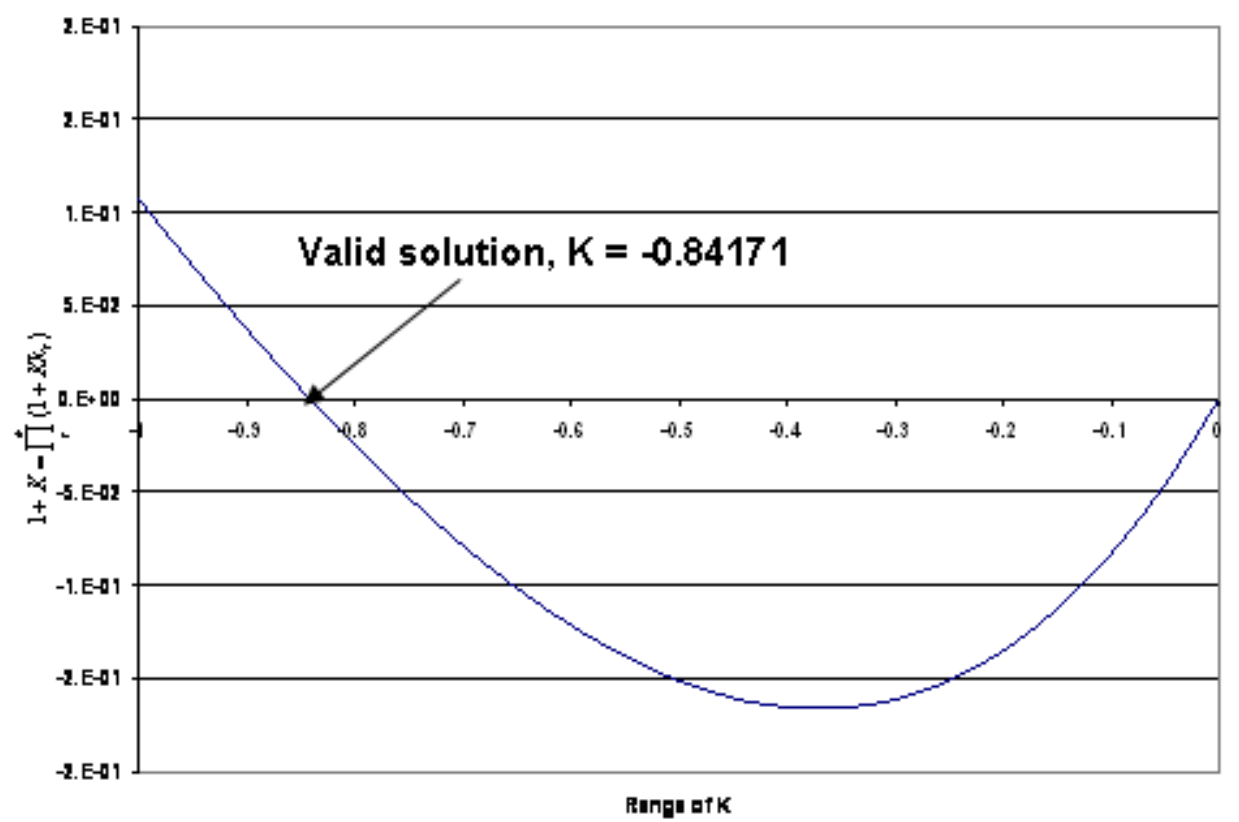

Figure $\mathrm{D}-1$. This shows a valid $K$ solution for 10 equal weights of $k=0.2$

It is interesting to note that there is little reason that a utility analysis on one level can not be the utility feed into a second tier method. Multiple layers based on different levels of attention to detail allow the MAUA methodology to scale from the atomistic to the astronomical. Solving for the utility function ( $u$ has become $u_{j}$ ) renders Equation (4). An example of one step upwards in a two stage analysis is shown in Equation (5). As can be seen, this can quickly become sufficiently complex and a third level is a natural extrapolation, but for simplicity and clarity only two levels are shown.

$$
\begin{aligned}
& u_{j}\left(x_{1}, x_{2} \ldots x_{i}\right)=\left(\left(\left(\prod_{i}^{n}\left(1+K k_{i} u_{i}\left(x_{i}\right)\right)\right)-1\right) / K\right) \\
& 1+K_{2} u_{\text {overall }}\left(x_{1}, x_{2} \ldots x_{i}\right)=\prod_{j}^{m}\left(1+K_{2} k_{j}\left(\left(\left(\prod_{i}^{n}\left(1+K k_{i} u_{i}\left(x_{i}\right)\right)\right)-1\right) / K\right)\right)
\end{aligned}
$$

MAUA methods are designed to be open-ended. They are generic functions that can be applied universally. Similarly to an artist with a blank canvas, a careful analyst could manipulate the analysis to render a result favorable to a specific ideology. The ultimate limitations of MAUA methods reside in the choice of utility functions, weights, and choice of attributes. As a result, no MAUA analysis should be taken at face value without a careful consideration for the specific attributes, weights, and utility functions applied. However, a standard set of attributes, weights, and utility functions, once determined, will render an objective, repeatable, and information-driven decision. 


\section{REFERENCES}

${ }^{1}$ Metcalf, R., Ragusa, J., Bean, R., "Safeguards Envelope: The First Steps,." 8th. Int. Conf. Facility Operations-Safeguards Interface. Portland, OR. 2008.

${ }^{2}$ Metcalf, R., Bevill, A., Charlton, W., Bean. R, "Safeguards Envelope: Previous Work and Examples." 49th Annual INMM National Conf. Nashville, TN. 2008.

${ }^{3}$ Ehinger, M. H. "Process Monitoring in I. Safeguards for Reprocessing Plants. - A Demonstration" CONF-8810269-1, 1988.

${ }^{4}$ Ehinger, M. H.. "Process Monitoring Concepts for Safeguards and Demonstrations at an Oak Ridge National Laboratory Facility.” N. Materials Management 15(1986):185-189.

${ }^{5}$ Burr, T.; Wangen, L. "Enhanced safeguards via solution monitoring."LA-13186-MS; ISPO390, Sep 1996.

${ }^{6}$ Burr, T. L.; Coulter, C. A.; Wangen, L. E. "Benchmark data for a large reprocessing plant for evaluation of advanced data analysis algorithms and safeguards system design." LA-13414MS, Feb 1998

${ }^{7}$ Burr, T. L.; Coulter, C. A.; Wangen, L. E. "Solution monitoring: Quantitative benefits to safeguards." Report: LA-UR-97-3467; IAEA-SM-351/43, 1997.

${ }^{8}$ Vilim, Richard B.. "Identification scheme combining first principle knowledge, neural networks, and the likelihood function." IEEE transactions on control systems technology 9.1 (2001):186-199.

${ }^{9}$ Garcia, Humberto E.. "Integrating physical modeling, neural computing, and statistical analysis for on-line process monitoring." Nuclear technology 141.1 (2003):69-77

${ }^{10}$ Garcia, Humberto E.. "Operational analysis and improvement of a spent nuclear fuel handling and treatment facility using discrete event simulation." Computers \& industrial engineering 38.2 (2000):235-249.

${ }^{11}$ H. Aigner, R. Binner, E. Kuhn. "International Target Values 2000 for Measurement Uncertainties in Safeguarding Nuclear Materials" IAEA, P. O. Box 100, A-1400 Wien, Austria. 2001.

${ }^{12}$ Close, D.A. MacArthur, D.W. Nicholas, N.J. " An early version of an information barrier "J. Nuclear Materials Management, v 31, n 1, Winter Fall, 2002.

${ }^{13}$ MacArthur, D., M. W. Johnson, and N. J. Nicholas. "Use of Information Barriers to Protect Classified Information." Proceedings of the 39th Annual Meeting of the Institute of Nuclear Materials Management. 1998.

${ }^{14}$ Menzel, J. H., "Safeguards Approach for Gas Centrifuge Type Enrichment Plants." J. of Nuclear Materials Management, Vol. XII, No. 4.1983.

${ }^{15}$ Wang, Xin, Wei, Thomas Y. C., Reifman, Jaques, and Tsoukalas, Lefteri. "Signal Trend Identification with FUZZY Method." IEEE International Conference on Information, Intelligence and Systems (ICIIS), Washington, DC, Nov. 1-3,1999 
${ }^{16}$ R.L. Keeney, and H. Raiffa, (1993) "Decisions with Multiple Objectives, Preferences and Value Tradeoffs", Cambridge University Press, Cambridge, UK.

${ }^{17}$ W. S. Charlton, R. F. LeBouf, C. Gariazzo, D. G. Ford, C. Beard, S. Landsberger and M. Whitaker, (2007) "Proliferation Resistance Assessment Methodology for Nuclear Fuel Cycles," Nuclear Technology," 157, 1.

${ }^{18}$ Chirayath,S., Metcalf, R., Ragusa, J., Nelson, P., “Assessment of Proliferation Resistance Requirements for Fast-Reactor Fuel-Cycle Facilities.” 8th. Int. Conf. Facility OperationsSafeguards Interface. Portland, OR. 2008.

${ }^{19}$ Metcalf, R., Ragusa, J., Bean, R., "Safeguards Envelope: The First Steps." 8th. Int. Conf. Facility Operations-Safeguards Interface. Portland, OR. 2008.

${ }^{20}$ Burr, T. L.; Coulter, C. A.; Wangen, L. E. "Benchmark data for a large reprocessing plant for evaluation of advanced data analysis algorithms and safeguards system design." LA-13414MS, Feb 1998

${ }^{21}$ Burr, T.; Jones, J.; Wangen, L. "Multivariate Diagnostics and Anomaly Detection For Nuclear Safeguards." LA-UR-94-2292; CONF-940748-44, 1994.

${ }^{22}$ Burr, T.; Wangen, L.; Mullen, M. "Authentication of Reprocessing Plant Safeguards Data Through Correlation Analysis." LA-12923-MS; ISPO-374, Apr 1995.

${ }^{23}$ R.L. Keeney, and H. Raiffa, (1993) "Decisions with Multiple Objectives, Preferences and Value Tradeoffs", Cambridge University Press, Cambridge, UK.

${ }^{24}$ Ham, W. L. "Selection of an Optimum Air Defense Weapon Package Using MAUM (MultiAttribute Utility Measurement)" Naval Postgraduate School, Monterey, CA., Jun 1983.

${ }^{25}$ Morikawa, Hidenori. "Optimal strengthening strategy on deteriorated concrete bridges based on multi-attribute utility approach." ざいりょう 49.2 (2000):181-186.

${ }^{26}$ Barbuceanu, Mihai, Lo, Wai-Kau, "Multi-Attribute Utility Theoretic negotiation architecture for electronic commerce" Proceedings of the Interantional Conference on Autonomous Agents, 2000.

${ }^{27}$ Zhai, Feng-Yong. "Estimation approach for dividable multi-attribute utility of housing via questionnaires of consumers' ordinal multi-criteria preferences." Ha Er Bin Gong Ye Da Xue Xue Bao ( Ying Wen Ban ) 11.5 (2004):519-523.

${ }^{28}$ R.L. Keeney, and H. Raiffa, (1993) "Decisions with Multiple Objectives, Preferences and Value Tradeoffs", Cambridge University Press, Cambridge, UK. 